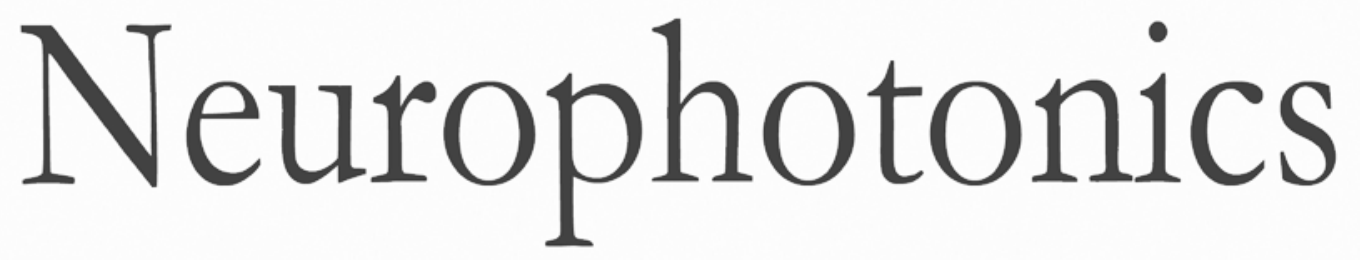

\title{
Pressure modulation algorithm to separate cerebral hemodynamic signals from extracerebral artifacts
}

Wesley B. Baker

Ashwin B. Parthasarathy

Tiffany S. Ko

David R. Busch

Kenneth Abramson

Shih-Yu Tzeng

Rickson C. Mesquita

Turgut Durduran

Joel H. Greenberg

David K. Kung

Arjun G. Yodh

\section{SPIE.}




\title{
Pressure modulation algorithm to separate cerebral hemodynamic signals from extracerebral artifacts
}

\author{
Wesley B. Baker, ${ }^{a, *}$ Ashwin B. Parthasarathy, ${ }^{a}$ Tiffany S. Ko, ${ }^{a}$ David R. Busch, ${ }^{a, b}$ \\ Kenneth Abramson, ${ }^{a}$ Shih-Yu Tzeng, ${ }^{a, c}$ Rickson C. Mesquita, ${ }^{d}$ Turgut Durduran, ${ }^{e}$ \\ Joel H. Greenberg, ${ }^{\prime}$ David K. Kung, ${ }^{9}$ and Arjun G. Yodh ${ }^{a}$ \\ aUniversity of Pennsylvania, Department of Physics and Astronomy, 3231 Walnut Street, Philadelphia, Pennsylvania 19104, United States \\ bChildren's Hospital of Philadelphia, Division of Neurology, 3401 Civic Center Boulevard, Philadelphia, Pennsylvania 19104, United States \\ 'National Cheng Kung University, Department of Photonics, No. 1, University Road, Tainan City 701, Taiwan \\ dUniversity of Campinas, Institute of Physics, 777 R. Sergio Buarque de Holanda, Campinas 13083-859, Brazil \\ eICFO-Institut de Ciències Fotòniques, Mediterranean Technology Park, Av. Carl Friedrich Gauss 3, Castelldefels (Barcelona) 08860, Spain \\ fUniversity of Pennsylvania, Department of Neurology, 3450 Hamilton Walk, Philadelphia, Pennsylvania 19104, United States \\ gHospital of the University of Pennsylvania, Department of Neurosurgery, 3400 Spruce Street, Philadelphia, Pennsylvania 19104, United States
}

\begin{abstract}
We introduce and validate a pressure measurement paradigm that reduces extracerebral contamination from superficial tissues in optical monitoring of cerebral blood flow with diffuse correlation spectroscopy (DCS). The scheme determines subject-specific contributions of extracerebral and cerebral tissues to the DCS signal by utilizing probe pressure modulation to induce variations in extracerebral blood flow. For analysis, the head is modeled as a two-layer medium and is probed with long and short source-detector separations. Then a combination of pressure modulation and a modified Beer-Lambert law for flow enables experimenters to linearly relate differential DCS signals to cerebral and extracerebral blood flow variation without a priori anatomical information. We demonstrate the algorithm's ability to isolate cerebral blood flow during a finger-tapping task and during graded scalp ischemia in healthy adults. Finally, we adapt the pressure modulation algorithm to ameliorate extracerebral contamination in monitoring of cerebral blood oxygenation and blood volume by near-infrared spectroscopy. ๑ 2015 Society of Photo-Optical Instrumentation Engineers (SPIE) [DOI: 10.1117/1.NPh.2.3.035004]
\end{abstract}

Keywords: diffuse correlation spectroscopy; near-infrared spectroscopy; functional brain imaging; cerebral blood flow monitoring; stroke.

Paper 15025R received May 12, 2015; accepted for publication Jul. 1, 2015; published online Aug. 4, 2015.

\section{Introduction}

Diffuse correlation spectroscopy ${ }^{1-5}$ (DCS) and diffuse optical or near-infrared spectroscopy ${ }^{6-13}$ (DOS/NIRS) are important optical techniques that employ near-infrared (NIR) light to measure cerebral blood flow, oxygen saturation, and total hemoglobin concentration continuously, noninvasively, and at the bedside. Furthermore, in combination, these measurements of blood flow and blood oxygenation provide access to the oxygen metabolic status of the brain. ${ }^{14-16}$

As might be anticipated, this information about cerebral blood flow, blood oxygenation, and oxygen metabolism has clinical value. All three parameters are important biomarkers for brain diseases, such as ischemic stroke. ${ }^{17,18}$ In fact, treatments for ischemic stroke, as well as other brain injuries and diseases, often aim to minimize neurological damage by maximizing perfusion to the brain lesion. ${ }^{19-21}$ Numerous treatment interventions for stroke are available, but variability in response to treatment has been observed, ${ }^{20-22}$ and an effective treatment for one patient may be ineffective or even harmful for another patient. Thus, rapid patient-specific assessment of treatment efficacy is a promising clinical application for DCS and DOS/NIRS. Potentially, DCS and DOS/NIRS measurements can enable detection

*Address all correspondence to: Wesley B. Baker, E-mail: wbb5uva@gmail .com of hemodynamic changes before new neurological symptoms emerge. ${ }^{2,23,24}$

Unfortunately, these optical techniques have limitations. A well-known drawback for optical monitoring of cerebral tissue is its significant sensitivity to blood flow and oxygenation in the extracerebral tissues (scalp and skull). ${ }^{25-29}$ Traditional diffuse optics analyses approximate the head as a homogeneous medium, e.g., no a priori anatomical knowledge is used. Homogeneous models ignore differences between extracerebral hemodynamics and cerebral hemodynamics in the brain, and because extracerebral blood flow and blood oxygenation are non-negligible, their responses contaminate DCS and DOS/NIRS signals. Specifically, extracerebral contributions can lead experimenters to incorrectly assign cerebral physiological responses. ${ }^{29-31}$

The DOS/NIRS community has, of course, developed approaches to ameliorate the extracerebral tissue problem. Timeseries analysis techniques, for example, use filtering schemes to minimize superficial tissue contamination in functional brain mapping measurements. ${ }^{26,27,29,32-38}$ An assumption that underlies these techniques is that superficial tissue contamination arises from systemic effects (e.g., heart rate) that do not correlate with cerebral response because systemic variations are typically damped by cerebral autoregulation. However, for numerous brain diseases, including ischemic stroke, cerebral autoregulation is impaired. ${ }^{39,40}$ In fact, many stroke treatment interventions

2329-423X/2015/\$25.00 @ 2015 SPIE 
are based on the notion of impaired cerebral autoregulation and are designed to increase cerebral blood flow through systemic mechanisms (e.g., increased blood pressure). Thus, it is preferable not to filter systemic components from the measured signals. In a different vein, computationally intense models have been explored to handle extracerebral heterogeneities directly, including layered models, ${ }^{41-49}$ Monte Carlo techniques in realistic geometries of the head,,$^{50-53}$ and imaging..$^{27,54-56}$ The complexity of these models, however, can make them impractical to implement for real-time monitoring. Further, these models often require a priori anatomical information about the patient's head, as well as knowledge about the optical properties of different tissue types.

In this contribution, we report on the implementation of a novel scheme for real-time cerebral monitoring with the twolayer model. The two-layer model in cerebral monitoring offers a compromise between simplicity and accuracy, ${ }^{57-65}$ it consists of a homogeneous superficial (extracerebral) layer above a homogeneous cerebral layer. The key to our new approach is to acquire DCS and DOS/NIRS measurements at multiple optical probe pressures and at multiple source-detector separations. Variations in probe pressure against the head induce variations in extracerebral hemodynamics, while cerebral hemodynamics remain constant. ${ }^{28}$ We will show how this information can be utilized to derive patient-specific analysis parameters that help to separate cerebral hemodynamics from extracerebral blood flow and oxygenation signals. For DCS measurements of blood flow, we employ the pressure modulation scheme and a twolayer modified Beer-Lambert framework for analysis. ${ }^{66}$ For DOS/NIRS measurements, we extend the two-layer modified Beer-Lambert formulation of Fabbri et al ${ }^{57}$ to include a pressure calibration stage prior to monitoring.

After describing the theory, we demonstrate the ability of this new measurement paradigm/algorithm to filter extracerebral contamination in simulations and in functional activation experiments on healthy adult humans. Ultimately, these developments should lead to improved accuracy in real-time monitoring of cerebral flow and oxygen metabolism.

\section{DCS and DOS/NIRS Monitoring (Homogeneous Tissue Model)}

Traditionally, diffuse optical monitoring utilizes homogeneous tissue models of the head, which we review first. The basic measurement geometry for diffuse optical monitoring consists of point illumination and point detection on the tissue surface; the distance between source and detector is $\rho$ [Fig. 1(a)]. DOS/NIRS is a static technique that measures slow (0.1 to $10 \mathrm{~s})$ variations in the detected light intensity induced by changes in tissue absorption $\left(\mu_{a}\right)$ and tissue scattering $\left(\mu_{s}^{\prime}\right)$. DCS is a qualitatively different dynamic light scattering technique that measures the rapid (e.g., microsecond scale) speckle light intensity fluctuations induced by red blood cell motion. DOS/NIRS measurements are most commonly analyzed with photon diffusion models ${ }^{67,68}$ and the modified Beer-Lambert law. ${ }^{69,70}$ Analogously, correlation diffusion models ${ }^{71,72}$ and the DCS modified Beer-Lambert law ${ }^{66}$ are readily employed for analysis of DCS measurements.

The modified Beer-Lambert law is arguably the most widely used homogeneous tissue model for analysis of DOS/NIRS measurements. ${ }^{69,70}$ The modified Beer-Lambert law relates changes in tissue optical properties to changes in continuouswave diffuse optical intensity measurements for light that has been multiply scattered in its trajectory through tissue (Fig. 1). Specifically, the measured difference in optical density between a perturbed state and a baseline state is related to tissue scattering and absorption differences of the corresponding perturbed and baseline states, i.e.,

$\Delta \mathrm{OD}=-\log \left(\frac{I}{I^{0}}\right) \approx L \Delta \mu_{a}+\frac{\mu_{a}^{0}}{\mu_{s}^{\prime 0}} L \Delta \mu_{s}^{\prime} \approx L \Delta \mu_{a}$.

Here, the tissue optical density is defined as the negative logarithm of the ratio of the detected and incident light intensities (time-averaged), i.e., $\mathrm{OD} \equiv-\log \left(I / I_{s}\right)$ for the perturbed state, and $\mathrm{OD}^{0} \equiv-\log \left(I^{0} / I_{s}\right)$ [Fig. 1(b)] for the baseline state; the incident light intensity, $I_{s}$, is assumed to remain constant. $\Delta \mathrm{OD} \equiv \mathrm{OD}-\mathrm{OD}^{0}, \Delta \mu_{a} \equiv \mu_{a}-\mu_{a}^{0}$, and $\Delta \mu_{s}^{\prime} \equiv \mu_{s}^{\prime}-\mu_{s}^{\prime 0}$ are the differential changes in tissue optical density, tissue absorption, and tissue reduced scattering, respectively, between a perturbed state $\left(\mathrm{OD}, \mu_{a}, \mu_{s}^{\prime}\right)$ and the baseline state $\left(\mathrm{OD}^{0}, \mu_{a}^{0}\right.$, $\left.\mu_{s}^{\prime 0}\right)$. The multiplicative factor, $L \equiv \partial \mathrm{OD}^{0} / \partial \mu_{a}$, is the differential pathlength, which is approximately the mean pathlength that diffusing photons travel through the medium from source to detector. ${ }^{70}$ For diffusive light transport, the differential pathlength can be computed using the solution to the photon
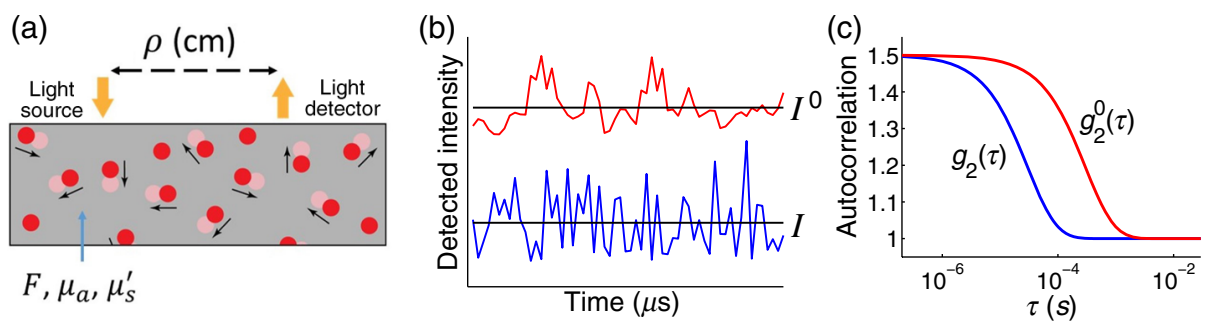

Fig. 1 (a) Schematic for a homogeneous, semi-infinite model of the head with a blood flow index, absorption coefficient, and reduced scattering coefficient of $F, \mu_{a}$, and $\mu_{s}^{\prime}$, respectively. The incident source intensity, $I_{s}$, is assumed to remain constant over time. Blood cell motion (e.g., red disks at time $t$ and light-red disks at time $t+\tau$ ) induces fast temporal fluctuations (i.e., speckle intensity fluctuations) in the detected light intensity on the time scale of microseconds, while absorption changes modify mean light intensities (e.g., averaged on time scales of milliseconds or greater). (b) Schematic of detected intensity fluctuations for a baseline tissue state (red curve) and a perturbed state from baseline with higher blood flow and absorption (blue curve). The horizontal black lines are the mean intensities for the two states, denoted as $I^{\circ}$ and $I$. The fast speckle intensity fluctuations in the two states are characterized by normalized intensity autocorrelation functions [i.e., $g_{2}^{0}(\tau), g_{2}(\tau)$ ]. (c) The decay of the intensity autocorrelation function curves is related to tissue blood flow. 
diffusion equation evaluated at the baseline tissue optical properties. ${ }^{69,70}$ For nondiffusive light transport, the differential pathlength can be computed using the solution to the radiative transport equation evaluated at the baseline tissue optical properties. $^{73}$ The modified Beer-Lambert law [Eq. (1)] is a first-order Taylor series expansion of the tissue optical density with respect to tissue absorption and tissue scattering. It is often reasonable to make the additional approximation that the scattering term in Eq. (1) is negligible compared to the absorption term; this approximation is reasonable because tissue scattering changes that accompany hemodynamic variations are often negligible, ${ }^{66}$ and because the multiplicative factor $\mu_{a}^{0} / \mu_{s}^{\prime 0}$ for many tissues is much less than one. Multispectral measurements of tissue absorption changes determined from Eq. (1) are then readily converted to estimates of the variation in tissue oxy-hemoglobin $(\mathrm{HbO})$ and deoxy-hemoglobin $(\mathrm{HbR})$ concentration using the well-known spectra of these molecules. ${ }^{5,74}$ The total hemoglobin concentration (HbT) is the sum of these two chromophore concentrations, and the tissue oxygen saturation $\left(\mathrm{StO}_{2}\right)$ is the ratio of oxy-hemoglobin to total hemoglobin: $\mathrm{HbT}=\mathrm{HbO}+\mathrm{HbR}$, $\mathrm{StO}_{2}=\mathrm{HbO} / \mathrm{HbT}$.

Equation (1) is valid for any homogeneous geometry, provided the correct differential pathlength is used. The differential pathlength depends on the source-detector separation $(\rho)$, the tissue geometry, and the baseline tissue optical properties $\left(\mu_{a}^{0}, \mu_{s}^{\prime 0}\right),{ }^{5,70}$ For the important special case of the semi-infinite homogeneous geometry [Fig. 1(a)], the differential pathlength is given by ${ }^{75}$

$L \approx \frac{3 \mu_{s}^{\prime 0} \rho^{2}}{2\left(\rho \sqrt{3 \mu_{a}^{0} \mu_{s}^{\prime 0}}+1\right)}$.

A drawback of the modified Beer-Lambert law is that it determines only the changes in hemoglobin concentration. For measurement of absolute oxy- and deoxy-hemoglobin concentrations, a photon diffusion model is commonly used. Formally, the detected light intensity is directly proportional to the photon diffusion equation Green's function for the appropriate tissue geometry, ${ }^{5}$ i.e., $\Phi(\rho)$, which depends on the tissue optical properties $\left(\mu_{a}, \mu_{s}^{\prime}\right)$. Note that the proportionality constant between the measured light intensity, $I(\rho)$, and the photon diffusion Green's function, $\Phi(\rho)$, is the light coupling coefficient to tissue for the source-detector pair. For semi-infinite homogeneous tissue, the continuous-wave photon diffusion equation Green's function is 5,76

$\Phi(\rho)=\frac{1}{4 \pi}\left[\frac{\exp \left(-r_{1} \sqrt{3 \mu_{a} \ell_{t r}}\right)}{r_{1}}-\frac{\exp \left(-r_{b} \sqrt{3 \mu_{a} \ell_{t r}}\right)}{r_{b}}\right]$.

Here, $\quad \ell_{t r}=1 /\left(\mu_{a}+\mu_{s}^{\prime}\right), \quad r_{1}=\left(\ell_{t r}^{2}+\rho^{2}\right)^{1 / 2}, \quad r_{b}=\left[\left(2 z_{b}+\right.\right.$ $\left.\left.\ell_{t r}\right)^{2}+\rho^{2}\right]^{1 / 2}$, and $z_{b}=2 \ell_{t r}\left(1+R_{\text {eff }}\right) /\left[3\left(1-R_{\text {eff }}\right)\right]$, where $R_{\text {eff }}$ is the effective reflection coefficient that accounts for the mismatch between the index of refraction of tissue $(n)$ and the index of refraction of the nonscattering medium bounding the tissue $\left(n_{\text {out }}\right)$, such as air. ${ }^{76}$ A standard approach for absolute tissue absorption monitoring in this geometry is to measure $I(\rho)$ at multiple source-detector separations and then obtain an estimate of $\mu_{a}$ by fitting these measured intensities to the semiinfinite Green's function solution [Eq. (3)]. Required inputs for this fit are the light coupling coefficients for each sourcedetector pair and the tissue reduced scattering coefficient, $\mu_{s}^{\prime}$.
Knowledge of the light coupling coefficients is typically obtained by calibration using a tissue phantom, ${ }^{77,78}$ and $\mu_{s}^{\prime}$ is often assumed. The assumption of $\mu_{s}^{\prime}$ is an obvious source of error for continuous-wave DOS/NIRS. In more complex frequency-domain ${ }^{79}$ and time-domain ${ }^{80}$ DOS/NIRS measurements, both $\mu_{a}$ and $\mu_{s}^{\prime}$ can be uniquely determined from a fitting of these measurements to their respective frequency-domain and time-domain Green's functions. ${ }^{5}$

DCS estimates blood flow by quantifying the fast speckle intensity fluctuations of multiply scattered coherent NIR light (with source coherence length $>5 \mathrm{~m}$ ) induced by red blood cell motion (Fig. 1). Specifically, the normalized intensity temporal autocorrelation function, $g_{2}(\tau) \equiv\langle I(t) I(t+\tau)\rangle /\langle I(t)\rangle^{2}$, is computed at multiple delay-times, $\tau$, where $I(t)$ is the detected light intensity at time $t$, and the angular brackets, \langle\rangle , represent time-averages. A DCS blood flow index, $F$, is derived from the decay of $g_{2}(\tau)$ [Fig. 1(c), discussed in more detail below]. The DCS blood flow index is directly proportional to tissue blood flow and has been successfully validated against a plethora of gold-standard techniques. ${ }^{1,81}$

In analogy to DOS/NIRS, a DCS modified Beer-Lambert law $^{66}$ relates differential changes in a DCS optical density, i.e., $\mathrm{OD}_{\mathrm{DCS}} \equiv-\log \left[g_{2}(\tau)-1\right]$, to differential changes in tissue blood flow index $(F)$, tissue absorption $\left(\mu_{a}\right)$, and tissue scattering $\left(\mu_{s}^{\prime}\right)$

$$
\begin{aligned}
\Delta \mathrm{OD}_{\mathrm{DCS}}= & -\log \left[\frac{g_{2}(\tau, \rho)-1}{g_{2}^{0}(\tau, \rho)-1}\right] \approx d_{F}(\tau) \Delta F+d_{a}(\tau) \Delta \mu_{a} \\
& +d_{s}(\tau) \Delta \mu_{s}^{\prime} .
\end{aligned}
$$

The multiplicative weighting factors $d_{F}(\tau) \equiv \partial \mathrm{OD}_{\mathrm{DCS}}^{0} / \partial F$, $d_{a}(\tau) \equiv \partial \mathrm{OD}_{\mathrm{DCS}}^{0} / \partial \mu_{a}$, and $d_{s}(\tau) \equiv \partial \mathrm{OD}_{\mathrm{DCS}}^{0} / \partial \mu_{s}^{\prime}$, can be estimated analytically or numerically using the correlation diffusion model applied to the appropriate geometry. ${ }^{66}$ These weighting factors are analogues of the differential pathlength in the modified Beer-Lambert law, but note that they also depend on delaytime, $\tau$. The DCS optical density is about equally sensitive to blood flow and tissue scattering changes, but is less sensitive to tissue absorption changes. ${ }^{66}$ Thus, if tissue scattering remains approximately constant, and the fractional absorption change is small compared to the blood flow change, then $\Delta \mathrm{OD}_{\mathrm{DCS}} \approx$ $d_{F}(\tau) \Delta F$. A system of equations is thus generated, i.e., one equation for each $\tau$; these equations can be solved for $\Delta F$ in a least squares sense (e.g., via the Moore-Penrose pseudoinverse technique $^{82}$ ). For the special case of the semi-infinite homogeneous geometry, the multiplicative weighting factor is given by ${ }^{66}$

$$
\begin{aligned}
d_{F}(\tau, \rho)= & \frac{6 \mu_{s}^{\prime 0}\left(\mu_{s}^{\prime 0}+\mu_{a}^{0}\right) k_{\mathrm{o}}^{2} \tau}{K^{0}(\tau)} \\
& \times\left\{\frac{\exp \left[-K^{0}(\tau) r_{1}^{0}\right]-\exp \left[-K^{0}(\tau) r_{b}^{0}\right]}{\exp \left[-K^{0}(\tau) r_{1}^{0}\right] / r_{1}^{0}-\exp \left[-K^{0}(\tau) r_{b}^{0}\right] / r_{b}^{0}}\right\},
\end{aligned}
$$

where $K^{0}(\tau)=\left[3 \mu_{a}^{0}\left(\mu_{a}^{0}+\mu_{s}^{\prime 0}\right)\left(1+2 \mu_{s}^{\prime 0} k_{\mathrm{o}}^{2} F^{0} \tau / \mu_{a}^{0}\right)\right]^{1 / 2}, \quad k_{\mathrm{o}}=$ $2 \pi n / \lambda$ is the magnitude of the light wave vector in the medium, and $r_{1}$ and $r_{b}$ are defined in Eq. (3).

The DCS modified Beer-Lambert law has a similar drawback to DOS/NIRS in that it only determines blood flow changes. To estimate the absolute blood flow index, $F$, a correlation diffusion approach is used. Formally, transport of the electric 
field $[\mathbf{E}(t)]$ autocorrelation function, $G_{1}(\tau) \equiv\left\langle\mathbf{E}^{*}(t) \cdot \mathbf{E}(t+\tau)\right\rangle$, is modeled by the correlation diffusion equation, ${ }^{71,72}$ which can be solved analytically or numerically for tissue geometries of interest. ${ }^{5,72}$ Tissue blood flow is ascertained by fitting the solution for the normalized electric field autocorrelation function, $g_{1}(\tau)=G_{1}(\tau) / G_{1}(\tau=0)$, to the measured normalized intensity autocorrelation function using the Siegert relation: ${ }^{83}$ $g_{2}(\tau)=1+\beta\left|g_{1}(\tau)\right|^{2}$, where $\beta$ is a constant determined primarily by experimental collection optics and source coherence.

For semi-infinite homogeneous tissue, the solution to the correlation diffusion equation is ${ }^{5,72}$

$G_{1}(\tau)=\frac{3}{4 \pi \ell_{t r}}\left\{\frac{\exp \left[-K(\tau) r_{1}\right]}{r_{1}}-\frac{\exp \left[-K(\tau) r_{b}\right]}{r_{b}}\right\}$,

where $K(\tau)$ is defined in Eq. (5), and $r_{1}, r_{b}$, and $\ell_{t r}$ are defined in Eq. (3).

A standard approach for blood flow monitoring with DCS in this geometry is to derive $g_{1}(\tau)$ from measurements of $g_{2}(\tau)$ via the Siegert relation. Then the semi-infinite correlation diffusion solution [Eq. (6)] is fit to $g_{1}(\tau)$ using a nonlinear minimization algorithm [e.g., Nelder-Mead simplex direct search ${ }^{84}$ implemented in MATLAB ${ }^{\circledR}$ (Mathworks, Natick, Massachusetts)], and an estimate of the blood flow index $(F)$ is obtained from the fit. As discussed above, these homogeneous head models do not distinguish cerebral hemodynamics from extracerebral hemodynamics, therefore, they are susceptible to extracerebral contamination.

\section{Probe Pressure Modulation Algorithm for Cerebral Blood Flow Monitoring with DCS}

Here we introduce our pressure modulation algorithm to separate cerebral blood flow from extracerebral artifacts. The scheme employs DCS measurements of the brain tissues at two probe pressures and two source-detector separations to reduce extracerebral contamination in cerebral blood flow monitoring. To distinguish extracerebral flow from cerebral flow, the head is modeled as a two-layer medium, ${ }^{57,58,63,72}$ and the sourcedetector separations are chosen such that detected light at the long separation (e.g., $\rho_{s}=3 \mathrm{~cm}$ ) travels through both layers, but detected light at the short separation (e.g., $\rho_{s}=1 \mathrm{~cm}$ ) is predominantly confined to the extracerebral layer [Fig. 2(a)]. Underlying this approach is our previous work, which showed that an increased probe pressure on the head is accompanied by a decrease in extracerebral flow; cerebral blood flow, however, is unchanged by probe pressure variation. ${ }^{28}$ Thus, the pressure-induced variation in the long-separation DCS signal [e.g., Fig. 2(b)] is due only to changes in extracerebral flow. This extracerebral flow change, in turn, is readily determined by the pressure-induced change measured in the short DCS separation signal [e.g., Fig. 2(c)], which can be analyzed using the semiinfinite medium approximation [Eq. (6)].

We will show that the subject-specific relative contributions of extracerebral and cerebral tissues to the long-separation DCS signal can be determined from the measured pressure-induced changes in the DCS signal at the long and short separations. Importantly, this patient-specific calibration with pressure modulation permits separation of the cerebral and extracerebral blood flow components in all subsequent measurements.

The results derived in Secs. 3.1 and 3.2 are for the special case of constant tissue absorption and tissue scattering. In practice, tissue scattering often remains roughly constant during hemodynamic changes. Further, for many cerebral processes, fractional changes in blood flow are substantially larger than fractional changes in tissue absorption. For example, for the finger-tapping functional response, ${ }^{59} F_{c} / F_{c}^{0} \sim 1.5, \mu_{a, c} / \mu_{a, c}^{0} \sim 1.1$ (at $\lambda=785 \mathrm{~nm}$ ); in this case, the flow contribution dominates the DCS signal change. ${ }^{66}$ We derive results for the general case wherein tissue absorption and scattering vary in Appendix A.

\subsection{Two-Layer Modified Beer-Lambert Laws for Flow at Long and Short Separations}

To filter contamination from extracerebral tissues in blood flow measurements of cerebral tissue, we use a two-layer modified Beer-Lambert formulation for blood flow based on the DCS measurement. ${ }^{66}$ In analogy with the DOS/NIRS modified Beer-Lambert law, ${ }^{69,70,85}$ a DCS optical density for the long and short source-detector separations at delay-time $\tau$ is defined as $\mathrm{OD}_{\mathrm{DCS}}^{\text {long }} \equiv-\log \left[g_{2}\left(\tau, \rho_{l}\right)-1\right]$ and $\mathrm{OD}_{\mathrm{DCS}}^{\text {short }} \equiv-\log \left[g_{2}\left(\tau, \rho_{s}\right)-1\right]$, respectively. Here, $g_{2}\left(\tau, \rho_{l}\right)$ and $g_{2}\left(\tau, \rho_{s}\right)$ are the measured long and short source-detector separation intensity autocorrelation functions with cerebral and extracerebral DCS blood flow indices $F_{c}$ and $F_{e c}$. Assuming constant tissue absorption and scattering, the two-layer modified Beer-Lambert equations for the long and short separations are ${ }^{66}$
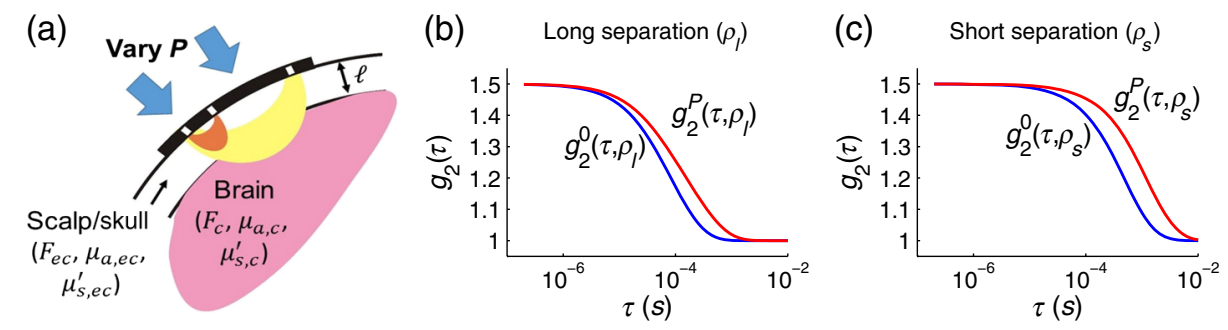

Fig. 2 (a) Two-layer tissue model of the head, which is composed of a semi-infinite bottom layer (i.e., corresponding to the cortical regions of the brain) with a distinct blood flow index, absorption coefficient, and reduced scattering coefficient of $F_{c}, \mu_{a, c}$, and $\mu_{s, c}^{\prime}$, respectively, and a superficial top layer (i.e., corresponding to extracerebral scalp and skull tissue) with thickness $\ell$, and distinct tissue properties denoted by $F_{e c}, \mu_{a, e c}$, and $\mu_{s, e c}^{\prime}$. The head is probed with a long source-detector separation, $\rho_{l}$ (yellow shading), and a short source-detector separation, $\rho_{s}$ (red shading), and the probe pressure against the head is varied. Increasing the probe pressure from $P^{0}$ (blue curves) to $P$ (red curves) induces a change in the diffuse correlation spectroscopy (DCS) signal $\left[g_{2}(\tau)\right]$ at both the long separation (b) and the short separation (c). These signal changes arise entirely from pressure-induced changes in extracerebral flow. ${ }^{28}$ 


$$
\begin{aligned}
\Delta \mathrm{OD}_{\mathrm{DCS}}^{\text {long }} & \equiv-\log \left[\frac{g_{2}\left(\tau, \rho_{l}\right)-1}{g_{2}^{0}\left(\tau, \rho_{l}\right)-1}\right] \\
& =d_{F, c}\left(\tau, \rho_{l}\right) \Delta F_{c}+d_{F, e c}\left(\tau, \rho_{l}\right) \Delta F_{e c}, \\
\Delta \mathrm{OD}_{\mathrm{DCS}}^{\text {short }} & \equiv-\log \left[\frac{g_{2}\left(\tau, \rho_{s}\right)-1}{g_{2}^{0}\left(\tau, \rho_{s}\right)-1}\right]=d_{F, e c}\left(\tau, \rho_{s}\right) \Delta F_{e c},
\end{aligned}
$$

where $g_{2}^{0}\left(\tau, \rho_{l}\right)$ and $g_{2}^{0}\left(\tau, \rho_{s}\right)$ are the baseline intensity autocorrelation functions at the long and short separations with cerebral and extracerebral DCS blood flow indices $F_{c}^{0}$ and $F_{e c}^{0}$ (note that the superscript 0 indicates baseline). The differential changes from baseline of cerebral and extracerebral blood flow are $\Delta F_{c} \equiv F_{c}-F_{c}^{0}$ and $\Delta F_{e c} \equiv F_{e c}-F_{e c}^{0}$, and the multiplicative weighting factors $d_{F, c}\left(\tau, \rho_{l}\right) \equiv \partial \mathrm{OD}_{\mathrm{DCS}}^{\text {long, } 0} / \partial F_{c}$ and $d_{F, e c}\left(\tau, \rho_{l}\right) \equiv$ $\partial \mathrm{OD}_{\mathrm{DCS}}^{\text {long, } 0} / \partial F_{e c}$ indicate the relative sensitivity of the long separation DCS optical density variation to cerebral versus extracerebral blood flow changes. For the short source-detector separation, the sensitivity of DCS optical density variation to extracerebral blood flow changes is $d_{F, e c}\left(\tau, \rho_{s}\right) \equiv \partial \mathrm{OD}_{\mathrm{DCS}}^{\text {short, } 0} /$ $\partial F_{e c}$. In Eq. (8), we have made the assumption that the short separation predominantly samples the extracerebral layer, and hence, the short separation signal is not sensitive to cerebral blood flow changes.

Solving the system of Eqs. (7) and (8) for $\Delta F_{c}$, we obtain

$$
\Delta F_{c}=\frac{1}{d_{F, c}\left(\tau, \rho_{l}\right)}\left[\Delta \mathrm{OD}_{\mathrm{DCS}}^{\text {long }}-\frac{d_{F, e c}\left(\tau, \rho_{l}\right)}{d_{F, e c}\left(\tau, \rho_{s}\right)} \Delta \mathrm{OD}_{\mathrm{DCS}}^{\text {short }}\right] .
$$

Notice that Eq. (9) is a linearized implementation of the twolayer head model (Fig. 2) that permits rapid monitoring of cerebral blood flow changes in real time. This implementation requires only one DCS delay-time $\tau$ for cerebral monitoring, but to ameliorate sensitivity to noise, multiple delay-times can also be used. In the latter case, Eq. (9) becomes a system of linear equations, i.e., one equation for each delay-time, which can be rapidly solved for $\Delta F_{c}$. Utilizing Eq. (9) in both the single and multiple delay-time implementations requires knowledge of $d_{F, c}\left(\tau, \rho_{l}\right)$ and $d_{F, e c}\left(\tau, \rho_{l}\right) / d_{F, e c}\left(\tau, \rho_{s}\right)$. A key result of this paper is that these weighting factors evaluated at the baseline tissue state can be estimated from initial DCS measurements acquired during probe pressure modulation against the head.

\subsection{Probe Pressure Modulation Calibration of DCS Weighting Factors}

A simple way to calibrate DCS for cerebral flow monitoring is to acquire long and short source-detector separation DCS measurements of the brain at two probe pressures (i.e., $P$ and $P^{0}$ ). It is not necessary to know the exact magnitudes of the probe pressures against the head. Further, the probe pressures need not be large, nor should patient comfort be compromised. The key for the modulation measurement is that the change in probe pressure from $P^{0}$ to $P$ should induce a change in extracerebral (i.e., scalp) blood flow. We recommend that the baseline probe pressure, $P^{0}$, be less than the venous blood pressure in the scalp, $P_{v}$, to ensure adequate scalp perfusion. Then, to decrease scalp blood flow for DCS calibration, the probe pressure needs to be increased to a value $P>P_{v}$ (Sec. 3.3) ${ }^{86}$ Thus, there are a broad range of pressures that can be used to calibrate DCS.

\subsubsection{Determination of $d_{F, e c}\left(\tau, \rho_{l}\right) / d_{F, e c}\left(\tau, \rho_{s}\right)$}

Recall that probe pressure modulation against the head affects extracerebral blood flow, but not cerebral blood flow, ${ }^{28}$ i.e., $\Delta F_{c}=0$ from probe pressure changes. Thus, the equations governing DCS measurements acquired at two different probe pressures, Eqs. (7) and (8), simplify to

$$
\begin{gathered}
\Delta \mathrm{OD}_{\mathrm{DCS}}^{\text {long, } P} \equiv-\log \left[\frac{g_{2}^{P}\left(\tau, \rho_{l}\right)-1}{g_{2}^{0}\left(\tau, \rho_{l}\right)-1}\right]=d_{F, e c}\left(\tau, \rho_{l}\right) \Delta F_{e c}^{P}, \\
\Delta \mathrm{OD}_{\mathrm{DCS}}^{\text {short } P} \equiv-\log \left[\frac{g_{2}^{P}\left(\tau, \rho_{s}\right)-1}{g_{2}^{0}\left(\tau, \rho_{s}\right)-1}\right]=d_{F, e c}\left(\tau, \rho_{s}\right) \Delta F_{e c}^{P} .
\end{gathered}
$$

Here $g_{2}^{P}\left(\tau, \rho_{l}\right)$ and $g_{2}^{P}\left(\tau, \rho_{s}\right)$ are the long and short separation intensity autocorrelation functions acquired at pressure $P$ wherein the cerebral and extracerebral flow indices are $F_{c}^{0}$ and $F_{e c}^{P}$, and $\Delta F_{e c}^{P} \equiv F_{e c}^{P}-F_{e c}^{0}$ is the pressure induced extracerebral flow change. Dividing Eq. (10) by Eq. (11) enables direct measurement of the ratio $d_{F, e c}\left(\tau, \rho_{l}\right) / d_{F, e c}\left(\tau, \rho_{s}\right)$, i.e.,

$$
\frac{d_{F, e c}\left(\tau, \rho_{l}\right)}{d_{F, e c}\left(\tau, \rho_{s}\right)}=\frac{\Delta \mathrm{OD}_{\mathrm{DCS}}^{\text {long }, P}}{\Delta \mathrm{OD}_{\mathrm{DCS}}^{\mathrm{short}, P}}
$$

Substituting Eq. (12) into Eq. (9), we obtain

$$
\Delta F_{c}=\frac{1}{d_{F, c}\left(\tau, \rho_{l}\right)}\left[\Delta \mathrm{OD}_{\mathrm{DCS}}^{\text {long }}-\frac{\Delta \mathrm{OD}_{\mathrm{DCS}}^{\text {long }, P}}{\Delta \mathrm{OD}_{\mathrm{DCS}}^{\mathrm{short}, P}} \Delta \mathrm{OD}_{\mathrm{DCS}}^{\text {short }}\right] .
$$

Notice that all of the terms within the square brackets above are derived from measurements. To the extent that the two-layer model (Fig. 2) accurately models the head, cerebral blood flow monitoring, as obtained from Eq. (13) (i.e., $\Delta F_{c}$ ), is not affected by extracerebral blood flow changes. The only assumptions used to derive Eq. (13) are that the probe pressure modulation has no effect on cerebral blood flow and that the tissue absorption and scattering remain constant. In Appendix A, Eq. (13) is extended to the more general case wherein tissue absorption and scattering can change, i.e., see Eq. (31). For accurate measurements of the absolute magnitude of the cerebral blood flow change, knowledge of $d_{F, c}\left(\tau, \rho_{l}\right)$ is also required.

\subsubsection{Determination of the weighting factor $d_{F, c}\left(\tau, \rho_{l}\right)$}

As we described previously, ${ }^{66}$ the multiplicative weighting factor $d_{F, c}\left(\tau, \rho_{l}\right)$ is readily computed numerically from the appropriate derivative of the two-layer correlation diffusion solution $\left(G_{1}\right){ }^{63,72}$

$$
\begin{aligned}
& d_{F, c}\left(\tau, \rho_{l}\right) \equiv \frac{\partial \mathrm{OD}_{\mathrm{DCS}}^{\text {long }, 0}}{\partial F_{c}}=2 \frac{\partial}{\partial F_{c}}\left\{-\log \left[G_{1}^{0}\left(\tau, \rho_{l}\right)\right]\right\}, \\
& \approx \frac{2}{\Delta F_{c}} \log \left[\frac{G_{1}\left(\tau, \rho_{l}, F_{c}^{0}-\Delta F_{c} / 2, F_{e c}^{0}, \mu_{a, c}^{0}, \mu_{a, e c}^{0}, \mu_{s, c}^{\prime 0}, \mu_{s, e c}^{\prime 0}, \ell\right)}{G_{1}\left(\tau, \rho_{l}, F_{c}^{0}+\Delta F_{c} / 2, F_{e c}^{0}, \mu_{a, c}^{0}, \mu_{a, e c}^{0}, \mu_{s, c}^{\prime 0}, \mu_{s, e c}^{\prime 0}, \ell\right)}\right],
\end{aligned}
$$

where $\Delta F_{c} / F_{c}^{0}=10^{-5}$. Evaluating Eq. (14) requires knowledge of the extracerebral layer thickness $(\ell)$, the baseline flow levels 
$\left(F_{c}^{0}, F_{e c}^{0}\right)$, and baseline tissue optical properties $\left(\mu_{a, c}^{0}, \mu_{a, e c}^{0}\right.$, $\left.\mu_{s, c}^{\prime 0}, \mu_{s, e c}^{\prime 0}\right)$.

Ideally, the extracerebral layer thickness is known from a priori anatomical information [e.g., from magnetic resonance imaging (MRI), computed tomography, x-ray, or ultrasound], and the baseline tissue optical properties are measured with concurrent frequency-domain or time-domain DOS/NIRS. ${ }^{61,62,64,87,88}$ Then estimates of $F_{c}^{0}$ and $F_{e c}^{0}$ are determined by simultaneously fitting the long-separation intensity autocorrelation curves measured at two pressures [i.e., $g_{2}^{0}\left(\tau, \rho_{l}\right)$ and $\left.g_{2}^{P}\left(\tau, \rho_{l}\right)\right]$ to the twolayer correlation diffusion solution. ${ }^{63,72}$ Important constraints used in this fit are that cerebral blood flow is the same for both probe pressures, i.e., $\Delta F_{c}^{P}=0$, and that the pressure-induced fractional extracerebral blood flow change, $\Delta F_{e c}^{P} / F_{e c}^{0}$, is determined from the short-separation measurements [i.e., $g_{2}^{0}\left(\tau, \rho_{s}\right)$, $\left.g_{2}^{P}\left(\tau, \rho_{s}\right)\right]$ via semi-infinite methods (Sec. 2). These constraints, facilitated by the pressure calibration data, render the nonlinear optimization in the fit more tractable and less sensitive to noise.

If it is not feasible to measure baseline tissue optical properties concurrently, then they can be assumed based on published cerebral/extracerebral measurements in the literature. ${ }^{25,60,64,89}$ Further, for some patients, a priori anatomical information may not be available; in this case, the extracerebral layer thickness, $\ell$, could be a third free parameter in the two-layer fit. Although fitting for three free parameters instead of two makes the fit more susceptible to noise and cross-talk, the fitting constraints provided by pressure calibration still enable reasonable estimates of $F_{c}^{0}, F_{e c}^{0}$, and $\ell$ to be derived.

An implicit assumption of this approach is that $\ell$ does not change with increasing probe pressure. To assess the validity of this assumption, we made a simple calculation of the scalp thickness variation using the Young's modulus for adipose tissue. ${ }^{90}$ Assuming a typical scalp thickness of $2 \mathrm{~mm},{ }^{91}$ the maximal probe pressure change of $25 \mathrm{~mm} \mathrm{Hg}$ induces a $0.4 \mathrm{~mm}$ decrease in scalp thickness, which is $<3 \%$ of a typical extracerebral layer thickness (e.g., $1.2 \mathrm{~cm}$ ). Such a small thickness change has a negligible effect on the DCS signal modeled by the two-layer model (i.e., $<1 \%$ ). Consequentially, the constant $\ell$ assumption is reasonable for the range of pressures employed.

As an aside, we have explored the utility of an alternative approach that uses short separation data to fit the semi-infinite correlation diffusion solution to $g_{2}^{0}\left(\tau, \rho_{s}\right)$ for $F_{e c}^{0}$ and to $g_{2}^{P}\left(\tau, \rho_{s}\right)$ for $F_{e c}^{P}$ (see Sec. 2). When using these absolute extracerebral flow indices as constraints in the two-layer fit to the long-separation data, only two free parameters $\left(F_{c}^{0}, \ell\right)$ remain to be fitted instead of three $\left(F_{c}^{0}, F_{e c}^{0}, \ell\right)$. However, the absolute extracerebral flow indices are sensitive to errors in extracerebral tissue optical properties, ${ }^{92}$ source-detector separation, head curvature, and heterogeneities within the scalp. Thus, from our experience, the first approach that utilizes robust fractional extracerebral flow change measurements is more reliable than the scheme that uses absolute extracerebral flow.

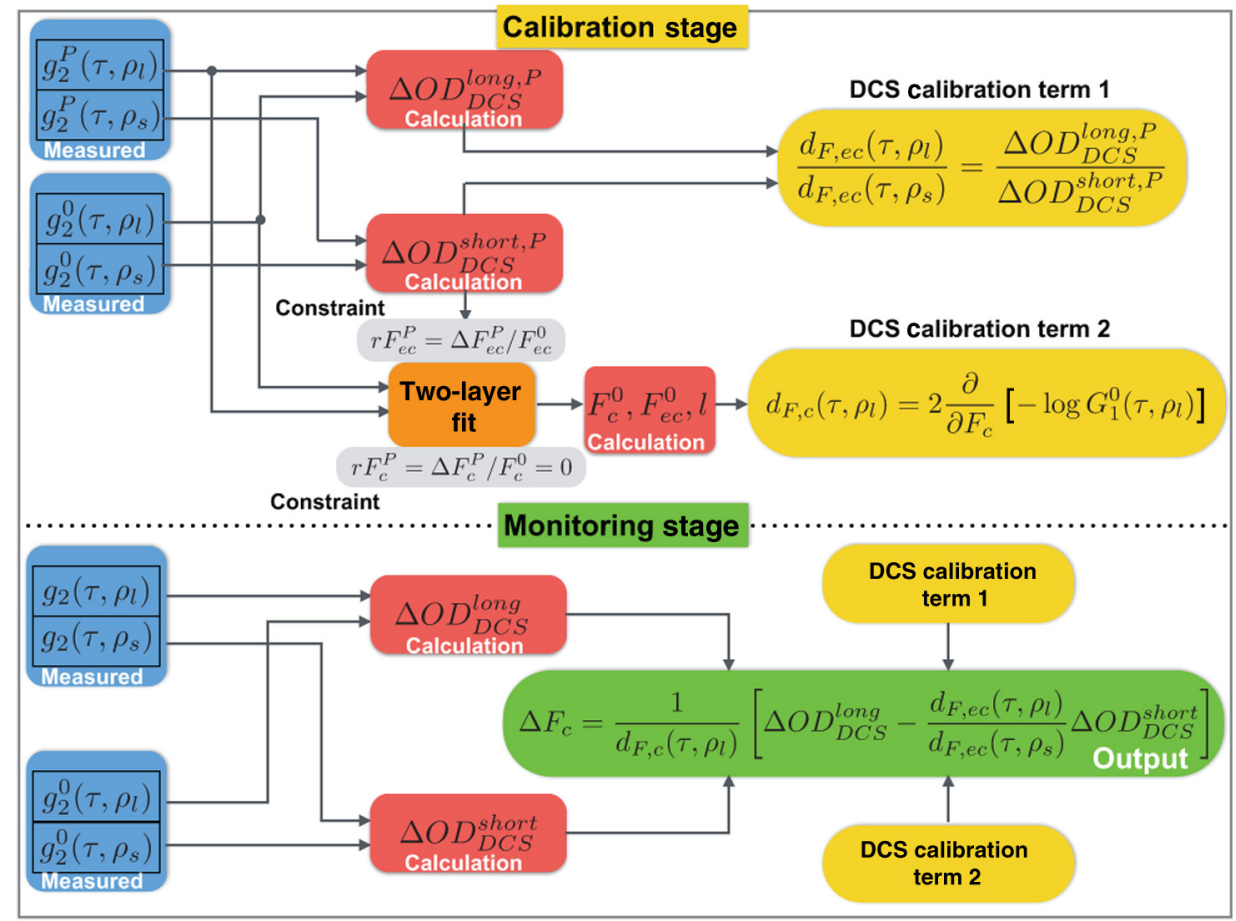

Fig. 3 Flow chart of probe pressure modulation algorithm for cerebral blood flow monitoring $\left(\Delta F_{c}\right)$ with DCS. In the "calibration stage," baseline long- and short-separation intensity autocorrelation functions measured at probe pressure $P^{0}\left[g_{2}^{0}\left(\tau, \rho_{l}\right), g_{2}^{0}\left(\tau, \rho_{s}\right)\right]$ and at probe pressure $P>P^{0}\left[g_{2}^{P}\left(\tau, \rho_{l}\right), g_{2}^{P}\left(\tau, \rho_{s}\right)\right]$ are used to evaluate "DCS calibration term 1" [Eq. (12)]. $F_{c}^{0}, F_{e c}^{0}$, and $\ell$ are extracted from a simultaneous fit of $g_{2}^{0}\left(\tau, \rho_{l}\right)$ and $g_{2}^{P}\left(\tau, \rho_{l}\right)$ to the two-layer correlation diffusion model (see Sec. 3.2.2), enabling numerical evaluation of "DCS calibration term 2" [Eq. (14)]. In the "monitoring stage," the DCS calibration terms 1 and 2 are employed to convert subsequent measurements of differential long- and short source-detector separation DCS optical density changes, i.e., $\Delta O D_{D C S}^{\text {long }}\left[\right.$ Eq. (7)] and $\triangle O D_{D C S}^{\text {short }}[E q$. (8)], to differential cerebral flow changes via Eq. (13). Note that the baseline used for the calibration stage and for the monitoring stage is the same. Finally, for this paper, we utilize delay times satisfying the limit $g_{2}^{0}\left(\tau, \rho_{l}\right)>1.25$ to solve Eq. (13). 


\subsection{Summary}

Figure 3 is a flow chart depicting the steps in the probe pressure modulation algorithm for filtering superficial tissue contamination in cerebral flow monitoring with DCS. In the "calibration stage" of the algorithm, intensity autocorrelation measurements at two probe pressures and two source-detector separations are used to compute the ratio $d_{F, e c}\left(\tau, \rho_{l}\right) / d_{F, e c}\left(\tau, \rho_{s}\right)$ ("DCS calibration term 1") and the long-separation weighting factor $d_{F, c}\left(\tau, \rho_{l}\right)$ ("DCS calibration term 2"). These calibration terms are then employed in the "monitoring stage" to permit the rapid estimation of cerebral flow changes $\left(\Delta F_{c}\right)$. To obtain the fractional cerebral flow change from baseline, we simply divide $\Delta F_{c}$ by the baseline cerebral flow index, $F_{c}^{0}$, obtained in the calibration stage.

In the calibration stage, a broad range of probe pressures will work, but not every set of probe pressures is useful. To understand why, note that scalp flow at baseline is driven by the blood pressure gradient $P_{a}-P_{v}$, where $P_{a}$ is the inlet arterial blood pressure supplying the scalp and $P_{v}$ is the outlet venous blood pressure draining the scalp. The probe pressure against the head controls the local extravascular tissue pressure, $P_{t}$. Increasing the probe pressure increases $P_{t}$, but if $P_{t}$ remains less than $P_{v}$, then the pressure gradient driving scalp flow is still approximately $P_{a}-P_{v}$, and scalp flow remains constant. ${ }^{86}$ When $P_{t}$ exceeds $P_{v}$, the outlet venous pressure increases to $P_{t}$ (e.g., via vasoconstriction) to keep vessels from collapsing, ${ }^{93}$ and the pressure gradient driving flow is $P_{a}-P_{t}$. At pressures $P_{t}>P_{a}$, vessels collapse and flow ceases. Especially for longterm flow monitoring, the baseline probe pressure $P^{0}$ should be less than $P_{v}$ to ensure adequate scalp perfusion. Then to temporarily change scalp blood flow for DCS calibration, the probe pressure $P$ must exceed $P_{v}$. For practical signal-to-noise ratio (SNR) levels obtained on the head, we found that a calibration probe pressure that exceeds $P_{v}$ by at least $5 \mathrm{~mm} \mathrm{Hg}$ ensures the pressure-induced change in the DCS signal is above the noise. In our measurement on a healthy adult subject, $P_{v}$ was $\sim 15 \mathrm{~mm} \mathrm{Hg}$, but remember that $P_{v}$ depends on several factors (e.g., blood pressure, posture).

\subsection{Correlation Noise Sensitivity}

The probe pressure modulation scheme depicted in Fig. 3 is a fast, patient-specific implementation of the two-layer model for cerebral flow monitoring. One difficulty that we must deal with concerns a high sensitivity to noise in the correlation measurement, especially at short delay-times. This sensitivity arises from the fact that correlation noise is largest at short delay-times, ${ }^{94}$ while the DCS optical density perturbations are typically small at short delay-times. Combined, these opposing trends with decreasing delay-time imply that the measured DCS optical density perturbations can be affected by both correlation noise and flow change signals under nonoptimal measurement conditions. To better understand these effects, consider a key step in the algorithm wherein DCS calibration term 1 [Eq. (12)] is computed and wherein a choice of delay-time must be made. The perturbation $\Delta \mathrm{OD}_{\mathrm{DCS}}^{\text {long } P}$ at short $\tau$ is less sensitive to the superficial blood flow changes induced by probe pressure modulation; this is because the rapid decay of the temporal autocorrelation signal at short $\tau$ is primarily due to long light paths that spend less time in superficial tissues. By contrast, the short light paths contribute to slow decay of the autocorrelation function (i.e., at long $\tau$ ). ${ }^{25,95}$
The choice of $\tau$ must, therefore, weight these effects. Computation of calibration term 1 at very short $\tau$ is prone to correlation noise and can lead to a significant systematic error in subsequent cerebral flow monitoring via Eq. (13).

Another noise-related issue that can arise is due to the fact that the autocorrelation signals at the long and short sourcedetector separations decay at substantially different rates. For example, at delay-times where the long-separation signal has decayed significantly, the short-separation signal has typically decayed much less. At these delay-times, the differences in short-separation decays induced by extracerebral flow changes are thus less pronounced than they are at longer delay-times, which means the measurement of $\Delta \mathrm{OD}_{\mathrm{DCS}}^{\text {short }}$ can be adversely affected by correlation noise.

We have identified an alternate approach for data analyses that helps to handle issues of correlation noise. The basic idea is to solve Eq. (7) directly for $\Delta F_{c}$ :

$\Delta F_{c}=\frac{1}{d_{F, c}\left(\tau, \rho_{l}\right)}\left[\Delta \mathrm{OD}_{\mathrm{DCS}}^{\text {long }}-d_{F, e c}\left(\tau, \rho_{l}\right) \Delta F_{e c}\right]$.

Here, $d_{F, c}\left(\tau, \rho_{l}\right)$ is given by Eq. (14), $d_{F, e c}\left(\tau, \rho_{l}\right) \equiv \partial \mathrm{OD}$ DCS short, $/$ $\partial F_{e c}$ is given by the extracerebral analogue of Eq. (14), and $\Delta F_{e c}$ is obtained from short-separation measurements via semi-infinite techniques (Sec. 2). Pressure variation is still used in the implementation of Eq. (15) via the two-layer fit for $F_{c}^{0}, F_{e c}^{0}$, and $\ell$ (Fig. 3). These baseline properties are inputs for determination of $d_{F, c}\left(\tau, \rho_{l}\right)$ and $d_{F, e c}\left(\tau, \rho_{l}\right)$. Finally, to derive the extracerebral flow change, we use the relation $\Delta F_{e c}=F_{e c}^{0} \times r F_{e c}$, where $r F_{e c} \equiv \Delta F_{e c} / F_{e c}^{0}$ is the fractional extracerebral flow change obtained from fitting the semi-infinite model to the short-separation autocorrelation curves. We have found that, on one hand, Eq. (15) is less sensitive to correlation noise, but, on the other hand, it is more reliant for the accuracy of the baseline tissue properties for filtering superficial tissue contamination. Thus, this approach is something that should be considered for analysis but may not be optimal.

\section{Pressure Modulation Algorithm for Cerebral Blood Flow Monitoring: Practical Example}

The purpose of this section is to provide an illustrative and explicit example of how the pressure modulation algorithm could be used in clinical practice. Here we consider cerebral blood flow monitoring during head-of-bed (HOB) position changes of stroke patients ${ }^{20,21}$ (Fig. 4). This study has already been carried out without pressure modulation. Briefly, to maximize perfusion at the ischemic core and the surrounding penumbra, flat HOB positioning [Fig. 4(b)] is often used in the clinic. In practice, changing the $\mathrm{HOB}$ angle from a baseline position of $30 \mathrm{deg}$. [Fig. 4(a)] to a flat position of 0 deg increases blood flow in the majority of patients. However, in a significant minority of patients $(25 \%)$, a paradoxical decrease in flow was observed. ${ }^{20,21}$ Thus, though the modulation scheme has not as yet been utilized in practice, optical cerebral flow monitoring with the probe pressure modulation algorithm holds potential for better characterization and optimization of HOB position on a patient-by-patient basis.

In the future, clinicians would carry out the following procedure. To determine cerebral flow changes induced by HOB position changes, the first step is a calibration stage that acquires long and short source-detector separation intensity 
(a)

Baseline HOB position

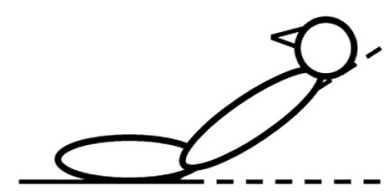

(b)

Flat $\mathrm{HOB}$ position (c)

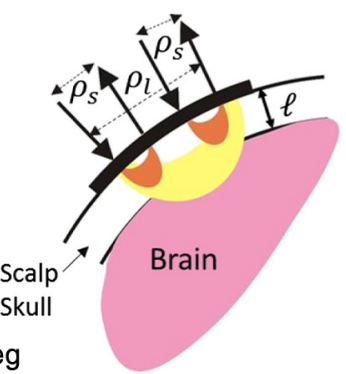

Fig. 4 Head-of-bed positioning at (a) the baseline condition of 30 deg and (b) the perturbed condition of 0 deg (flat). (c) Schematic of two-layer geometry of the head probed with a long separation, $\rho_{l}$, and two short separations, $\rho_{s}$. The downward and upward pointing arrows indicate DCS source and detector positions, respectively.

autocorrelation measurements at the $30 \mathrm{deg} \mathrm{HOB}$ position with a probe pressure $P$ (e.g., $P=20 \mathrm{~mm} \mathrm{Hg}$ ) applied against the scalp, i.e., $g_{2}^{P}\left(\tau, \rho_{l}\right), g_{2}^{P}\left(\tau, \rho_{s}\right)$. The next step (step 2 ) of the calibration process is to decrease the probe pressure against the scalp to $P^{0}$ (e.g., $P^{0}=5 \mathrm{~mm} \mathrm{Hg}$ ), and at this new probe pressure $\left(P^{0}\right)$ and the same $30 \mathrm{deg}$ HOB position, the clinician should acquire a second set of long and short source-detector separation intensity autocorrelation measurements, i.e., $g_{2}^{0}(\tau$, $\left.\rho_{l}\right), g_{2}^{0}\left(\tau, \rho_{s}\right)$. Using these two sets of measurements, we then compute DCS calibration terms 1 and 2 from Fig. 3. These calibration terms will then be employed in the monitoring stage to determine cerebral flow changes from baseline (Fig. 3).

Continuing with our example, we change the HOB position from 30 to $0 \mathrm{deg}$, and acquire $g_{2}\left(\tau, \rho_{l}\right)$ and $g_{2}\left(\tau, \rho_{s}\right)$, which are the long and short source-detector separation autocorrelation measurements at the $0 \mathrm{deg} \mathrm{HOB}$ position (i.e., the perturbed state). The cerebral flow change due to the HOB change, i.e., $\Delta F_{c} \equiv F_{c}(0 \mathrm{deg})-F_{c}^{0}(30 \mathrm{deg})$, is given by Eq. (13).

To the extent that the two-layer model accurately models the head, the cerebral flow changes calculated in this manner will be less sensitive to blood flow in superficial (extracerebral) tissues. Of course, the two-layer model approximates the head as a spatially uniform superficial tissue layer above a semi-infinite cerebral layer. In practical measurements of the head, interference from superficial tissues in cerebral monitoring is sometimes spatially inhomogeneous across the surface of the scalp. ${ }^{33,96}$ One way to reduce error from these superficial heterogeneities is to probe the superficial tissue volume above the cerebral region of interest with multiple short source-detector separations, as shown in Fig. 4(c). It is straightforward to extend the probe pressure modulation algorithm to handle multiple short separations. In our measurements (to be discussed below), we followed the steps outlined in Fig. 3 for each short separation separately in order to obtain an estimate of the cerebral flow change. We then averaged the two estimates of $\Delta F_{c}$ obtained from the two short separations. This averaging was not strictly necessary in our measurements, since we generally found the short-separation signals to be the same within our signal-to-noise.

\section{Probe Pressure Modulation Algorithm for Oxygenation Monitoring with Diffuse Optical or Near-Infrared Spectroscopy}

In Sec. 3, we developed a probe pressure modulation paradigm for DCS that filters contamination from superficial tissues in cerebral blood flow measurements. An analogous probe pressure modulation scheme can be used to calibrate continuouswave DOS/NIRS for monitoring cerebral oxy-hemoglobin
$\left(\mathrm{HbO}_{c}\right)$ and deoxy-hemoglobin $\left(\mathrm{HbR}_{c}\right)$ concentrations. This scheme also employs a two-layer modified Beer-Lambert framework.

\subsection{Two-Layer Modified Beer-Lambert Laws for Absorption at Long and Short Separations}

DOS/NIRS measurements of light intensity are made at a long source-detector separation, $I\left(\rho_{l}\right)$, and a short source-detector separation, $I\left(\rho_{s}\right)$. Using a two-layer model of the head, the DOS/NIRS two-layer modified Beer-Lambert law analogues of Eqs. (7) and (8) are $\mathrm{ar}^{57,85}$

$$
\Delta \mathrm{OD}^{\text {long }} \equiv-\log \left[\frac{I\left(\rho_{l}\right)}{I^{0}\left(\rho_{l}\right)}\right]=L_{c}\left(\rho_{l}\right) \Delta \mu_{a, c}+L_{e c}\left(\rho_{l}\right) \Delta \mu_{a, e c},
$$

$\Delta \mathrm{OD}^{\text {short }} \equiv-\log \left[\frac{I\left(\rho_{s}\right)}{I^{0}\left(\rho_{s}\right)}\right]=L_{e c}\left(\rho_{s}\right) \Delta \mu_{a, e c}$.

The cerebral and extracerebral tissue absorption and reduced scattering coefficients that give rise to the measured intensities $I\left(\rho_{l}\right)$ and $I\left(\rho_{s}\right)$ are $\mu_{a, c}, \mu_{a, e c}, \mu_{s, c}^{\prime}$, and $\mu_{s, e c}^{\prime}$, respectively. Similarly, at the baseline measured intensities $I^{0}\left(\rho_{l}\right)$ and $I^{0}\left(\rho_{s}\right)$, the baseline cerebral and extracerebral tissue absorption and reduced scattering coefficients are $\mu_{a, c}^{0}, \mu_{a, e c}^{0}, \mu_{s, c}^{\prime 0}$, and $\mu_{s, e c}^{\prime 0}$, respectively. The differential changes of cerebral and extracerebral absorption from baseline are $\Delta \mu_{a, c} \equiv \mu_{a, c}-\mu_{a, c}^{0}$ and $\Delta \mu_{a, e c} \equiv \mu_{a, e c}-\mu_{a, e c}^{0}$; for simplicity, tissue scattering will be assumed to be constant in this treatment. Finally, the partial pathlengths $L_{c}\left(\rho_{l}\right) \equiv \partial \mathrm{OD}^{\text {long }, 0} / \partial \mu_{a, c}, L_{e c}\left(\rho_{l}\right) \equiv \partial \mathrm{OD}^{\text {long, }, 0} / \partial \mu_{a, e c}$, and $L_{e c}\left(\rho_{s}\right) \equiv \partial \mathrm{OD}^{\text {short }, 0} / \partial \mu_{a, e c}$ are the mean pathlengths that the detected light travels through the cerebral $(c)$ and extracerebral (ec) layers. ${ }^{57,58,85}$ If the short source-detector separation is comparable to the extracerebral layer thickness, it is reasonable to assume that detected light from the short separation does not sample the brain, and consequentially, $L_{c}\left(\rho_{s}\right)=0$ and $L_{e c}\left(\rho_{s}\right)$ is approximately the semi-infinite differential pathlength given by Eq. (2).

Following steps analogous to those outlined for flow monitoring in Sec. 3, probe pressure modulation can be used to calibrate DOS/NIRS for cerebral absorption monitoring (see Appendix B), i.e.,

$$
\Delta \mu_{a, c}=\frac{1}{L_{c}\left(\rho_{l}\right)}\left[\Delta \mathrm{OD}^{\text {long }}-\frac{\Delta \mathrm{OD}^{\text {long }, P}}{\Delta \mathrm{OD}^{\text {short }, P}} \Delta \mathrm{OD}^{\text {short }}\right] .
$$


Here, $\Delta \mathrm{OD}^{\text {long, } P}$ and $\Delta \mathrm{OD}^{\text {short }, P}$ are the long and short separation changes in optical density induced by the probe pressure change $\Delta P=P-P^{0}$, and $L_{c}\left(\rho_{l}\right)$ is calculated by numerically computing the derivative of the continuous-wave two-layer photon diffusion Green's function, $\Phi\left(\rho_{l}\right),{ }^{44,88}$ evaluated with the baseline tissue optical properties:

$$
\begin{aligned}
L_{c}\left(\rho_{l}\right) & =\frac{\partial}{\partial \mu_{a, c}}\left\{-\log \left[\Phi^{0}\left(\rho_{l}\right)\right]\right\} \\
& \approx \frac{1}{\Delta \mu_{a, c}} \log \left[\frac{\Phi\left(\rho_{l}, \mu_{a, c}^{0}-\Delta \mu_{a, c} / 2, \mu_{a, e c}^{0}, \mu_{s, c}^{\prime 0}, \mu_{s, e c}^{\prime 0}, \ell\right)}{\Phi\left(\rho_{l}, \mu_{a, c}^{0}+\Delta \mu_{a, c} / 2, \mu_{a, e c}^{0}, \mu_{s, c}^{\prime 0}, \mu_{s, e c}^{\prime 0}, \ell\right)}\right],
\end{aligned}
$$

where $\Delta \mu_{a, c} / \mu_{a, c}^{0}=10^{-5}$. The Green's function $\Phi\left(\rho_{l}\right)$ can be evaluated using the analytical two-layer solution, or it can also be evaluated numerically using Monte Carlo techniques. ${ }^{46}$

Figure 5 is a flow chart summarizing the DOS pressure modulation algorithm for monitoring cerebral absorption changes. Note that this algorithm can be generalized for monitoring with multiple short separations in a manner exactly analogous to that described in Sec. 4.

\subsection{Multispectral Diffuse Optical or Near-Infrared Spectroscopy Cerebral Absorption Measurements Enable Hemoglobin Monitoring}

The cerebral tissue absorption coefficient depends linearly on the concentrations of tissue chromophores. With NIR light, changes in cerebral absorption predominantly arise from changes in cerebral oxygenated hemoglobin $\left(\mathrm{HbO}_{c}\right)$ and deoxygenated hemoglobin $\left(\mathrm{HbR}_{c}\right)$ concentrations, such that ${ }^{5}$

$$
\Delta \mu_{a, c}\left(\rho_{l}, \lambda\right) \approx \varepsilon_{\mathrm{HbO}}(\lambda) \Delta \mathrm{HbO}_{c}+\varepsilon_{\mathrm{HbR}}(\lambda) \Delta \mathrm{HbR}_{c} .
$$

Here, $\varepsilon_{\mathrm{HbO}}(\lambda)$ and $\varepsilon_{\mathrm{HbR}}(\lambda)$ are wavelength-dependent extinction coefficients for oxygenated hemoglobin and deoxygenated hemoglobin, which are both known and tabulated as a function of wavelength $\lambda{ }^{74}$ and $\Delta \mathrm{HbO}_{c}$ and $\Delta \mathrm{HbR}_{c}$ are differential changes in cerebral oxygenated and deoxygenated hemoglobin concentration from baseline. For multispectral cerebral absorption monitoring with Eq. (18), Eq. (20) becomes a system of equations, i.e., one equation for each wavelength, which can then be solved for $\Delta \mathrm{HbO}_{c}$ and $\Delta \mathrm{HbR}_{c}$. A minimum of two wavelengths is required to solve for these two chromophores.

Finally, the baseline cerebral hemoglobin concentrations $\mathrm{HbO}_{c}^{0}$ and $\mathrm{HbR}_{c}^{0}$ can be calculated from multispectral measurements of $\mu_{a, c}^{0}(\lambda)$, which in turn enables the computation of cerebral tissue oxygen saturation, $\mathrm{StO}_{2, c} .{ }^{5}$

$$
\mathrm{StO}_{2, c}=\frac{\mathrm{HbO}_{c}^{0}+\Delta \mathrm{HbO}_{c}}{\mathrm{HbO}_{c}^{0}+\mathrm{HbR}_{c}^{0}+\Delta \mathrm{HbO}_{c}+\Delta \mathrm{HbR}_{c}} .
$$

As many researchers have discussed, combining DOS/NIRS measurements of $\mathrm{StO}_{2, c}$ with DCS measurements of cerebral blood flow $\left(F_{c}\right)$ permits monitoring of cerebral oxygen metabolism. ${ }^{14,15}$

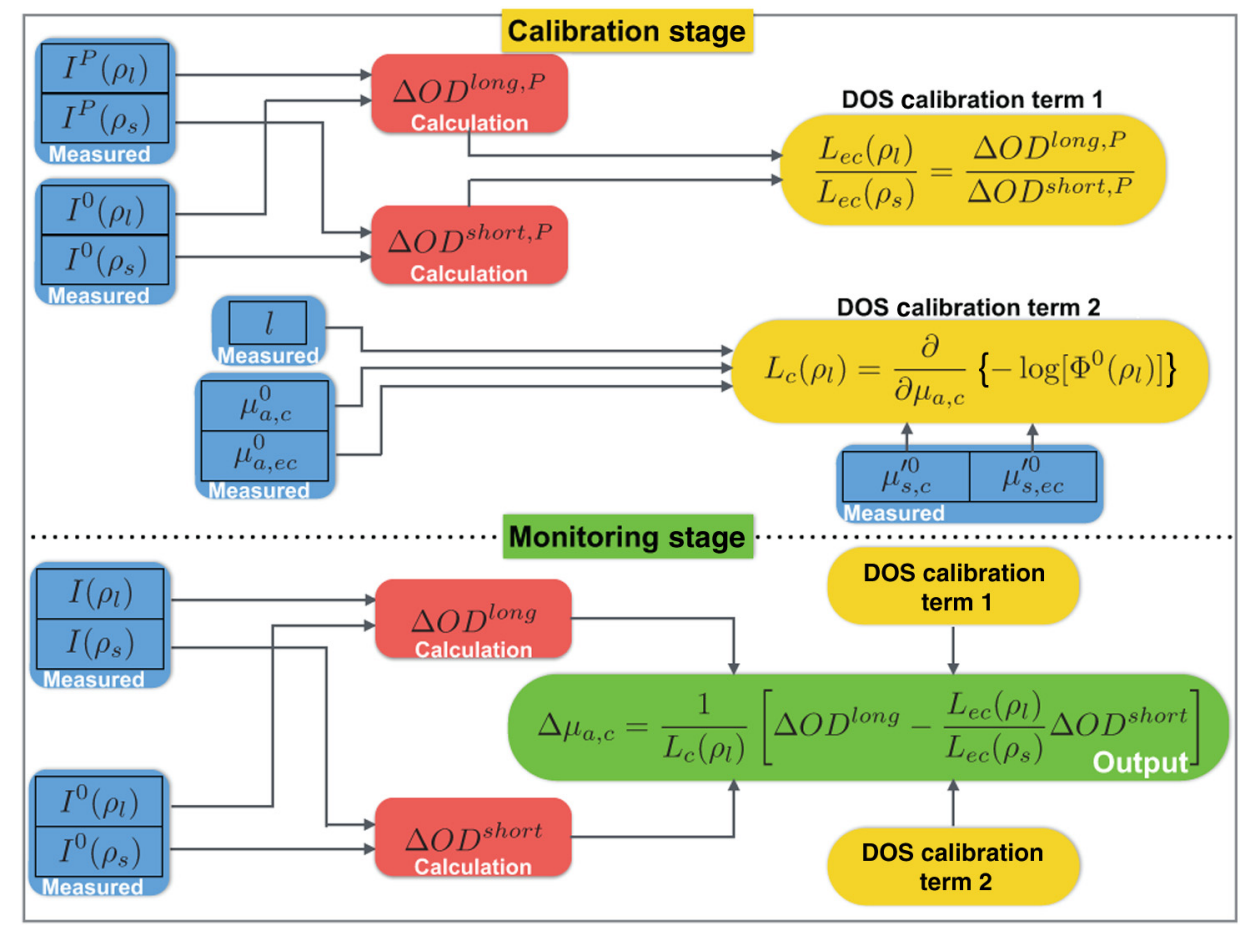

Fig. 5 Flow chart of probe pressure modulation algorithm for cerebral tissue absorption monitoring $\left(\Delta \mu_{a, c}\right)$ with diffuse optical or near-infrared spectroscopy (DOS/NIRS). In the "calibration stage," baseline long and short source-detector separation intensities measured at probe pressure $P^{0}\left[I^{0}\left(\rho_{l}\right), I^{0}\left(\rho_{s}\right)\right]$ and at probe pressure $P>P^{0}\left[I^{P}\left(\rho_{I}\right), I^{P}\left(\rho_{s}\right)\right]$ are used to evaluate "DOS calibration term 1." "DOS calibration term 2" is the numerical evaluation of $L_{c}\left(\rho_{l}\right)$ [Eq. (19)], which requires knowledge of the baseline tissue optical properties and the extracerebral layer thickness $(\ell)$. Ideally, these baseline tissue properties are measured (see Sec. 3.2.2). In the "monitoring stage," the DOS calibration terms 1 and 2 are employed to convert subsequent measurements of differential long and short source-detector separation optical density changes, i.e., $\Delta O D^{\text {long }}$ [Eq. (16)] and $\Delta O D^{\text {short }}$ [Eq. (17)], to differential cerebral absorption changes via Eq. (18). Note that the baseline used for the calibration stage and for the monitoring stage is the same. 


\section{Experimental Methods}

We have successfully applied the pressure modulation algorithms described to both simulated data with noise and to in vivo measurements in healthy adult volunteers that measure cerebral hemodynamic changes. Each of the two adults measured provided written consent, and all protocols/procedures were approved by the institutional review board at the University of Pennsylvania. One adult sat comfortably while we acquired data at several different probe pressures against the scalp, i.e., in order to induce graded scalp ischemia. As discussed above, probe pressure modulation changes extracerebral flow, but cerebral flow remains constant. ${ }^{28}$ The second adult was asked to do a finger-tapping task, which induces a localized cerebral blood flow increase in the motor cortex along with a more global extracerebral flow increase (from systemic effects). ${ }^{13,27,29}$ The instrumentation used for the in vivo measurements are described in Appendix C, and the measurement protocols are explained in Secs. 6.2 and 6.3. We first discuss the generation of simulated data.

\subsection{Simulated Data}

For light wavelength $\lambda=785 \mathrm{~nm}$, we generated simulated intensity autocorrelation functions (DCS) and light intensities (DOS/NIRS) at source-detector separations of $\rho_{l}=3 \mathrm{~cm}$ and $\rho_{s}=0.7 \mathrm{~cm}$ for two types of hemodynamic perturbations. Simulated DCS data sets were obtained for the special cases of (1) varying cerebral flow while extracerebral flow remains constant and (2) varying extracerebral flow while cerebral flow remains constant. Similarly, simulated DOS/NIRS intensity data sets were obtained for the special cases of (1) varying cerebral absorption while extracerebral absorption remains constant and (2) varying extracerebral absorption while cerebral absorption remains constant. The simulated intensity autocorrelation functions were generated from two-layer solutions of the correlation diffusion equation ${ }^{63,72}$ with added correlation noise. ${ }^{94}$ Simulated DOS/NIRS intensities were generated from twolayer solutions of the photon diffusion equation 44,88 with added Gaussian noise.

Baseline tissue optical properties and tissue blood flow levels in the simulated data were chosen to be representative of the head, i.e., $\mu_{a, c}^{0}=0.16, \mu_{a, e c}^{0}=0.12, \mu_{s, c}^{\prime 0}=6, \mu_{s, e c}^{\prime 0}=10 \mathrm{~cm}^{-1}$; $F_{c}^{0}=1.4 \times 10^{-8}, \quad F_{e c}^{0}=1.4 \times 10^{-9} \mathrm{~cm}^{2} / \mathrm{s} ; \ell=1.2 \mathrm{~cm} \quad$ [see Fig. 2; optical properties from Ref. 60, extracerebral flow from Ref. 28, cerebral to extracerebral flow ratio from Ref. 97, and the extracerebral layer thickness from averaging across MRI measurements in nine adult volunteers (Durduran et al., unpublished)]. In the DCS simulations, tissue optical properties remained constant, and the added correlation noise was derived from a correlation noise model ${ }^{94}$ evaluated at DCS photon count rates of 50 and $100 \mathrm{kHz}$ for the long and short source-detector separations, respectively, and an integration time of $2.5 \mathrm{~s}$. The DCS signals for each pair of cerebral and extracerebral flow levels in the data sets were obtained by averaging across $N=100$ simulated autocorrelation functions with noise. Finally, to simulate an increased probe pressure during the calibration stage of the measurement (Fig. 3), the extracerebral blood flow was decreased by $30 \%$ from baseline.

In the DOS/NIRS simulations, tissue optical scattering remained constant, and the added light intensity noise was derived from a Gaussian noise model ( $\mathrm{SNR} \equiv \mu / \sigma=100)$. The DOS/NIRS signal for each pair of cerebral and extracerebral tissue absorption coefficients in the data sets was obtained by averaging across $N=100$ simulated intensities, and the extracerebral tissue absorption was decreased by $15 \%$ from baseline to simulate the increased probe pressure during the calibration stage (Fig. 5).

\subsection{Graded Scalp Ischemia Protocol}

First, absolute baseline optical properties over the subject's left forehead were measured with a multiple-distance frequencydomain technique. ${ }^{77,78}$ Specifically, a commercial frequencydomain ISS Imagent (ISS Medical, Champaign, Illinois) was connected to a multiple-distance probe (ISS Medical, $\rho=2$, $2.5,3,3.5 \mathrm{~cm})$. Prior to the forehead measurement, the instrument was first calibrated on a solid silicon phantom (ISS Medical) with known optical properties. ${ }^{77,78}$ We used these measurements of the bulk average optical properties over the sampled tissue volume for both the cerebral and extracerebral layers.

Then as the subject sat comfortably, an optical probe [Fig. 4(c)] with one long separation $\left(\rho_{l}=3.0 \mathrm{~cm}\right)$ and two short separations $\left(\rho_{s}=1.0 \mathrm{~cm}\right)$ was placed on the subject's left forehead and secured with a blood pressure arm cuff (Soma Technology, Bloomfield, Connecticut) wound around the head [Fig. 6(a)]. The pressure cuff was inflated and maintained at the desired air pressure with a Zimmer ATS-1500 tourniquet system (Zimmer Inc., Warsaw, Indiana). DCS measurements were acquired at five different probe pressures against the scalp (i.e., five different extracerebral blood flow levels) ranging from 15 to $40 \mathrm{~mm} \mathrm{Hg}$ [Fig. 6(b)]. Here, the calculation of cerebral flow involved averaging over the measured signals as described in Sec. 4.

\subsection{Finger-Tapping Protocol}

Throughout the finger-tapping measurement, the subject lay supine on a bed. As with the scalp ischemia measurement (a)

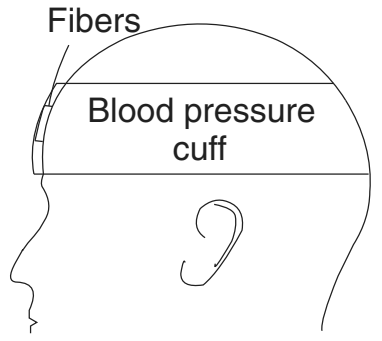

(b)

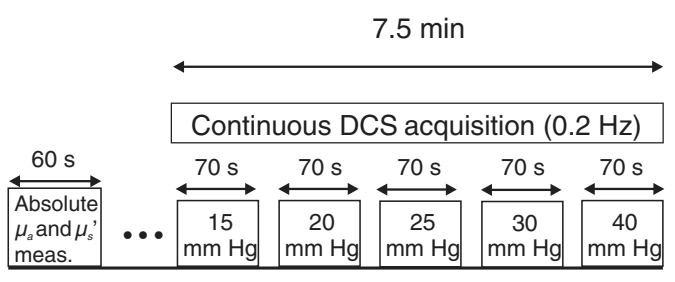

Fig. 6 Cerebral blood flow monitoring during graded scalp ischemia. (a) A blood pressure cuff wound around the head was used to uniformly adjust the pressure of the optical probe against the forehead. (b) DCS measurements were made at five different probe pressures against the scalp. 


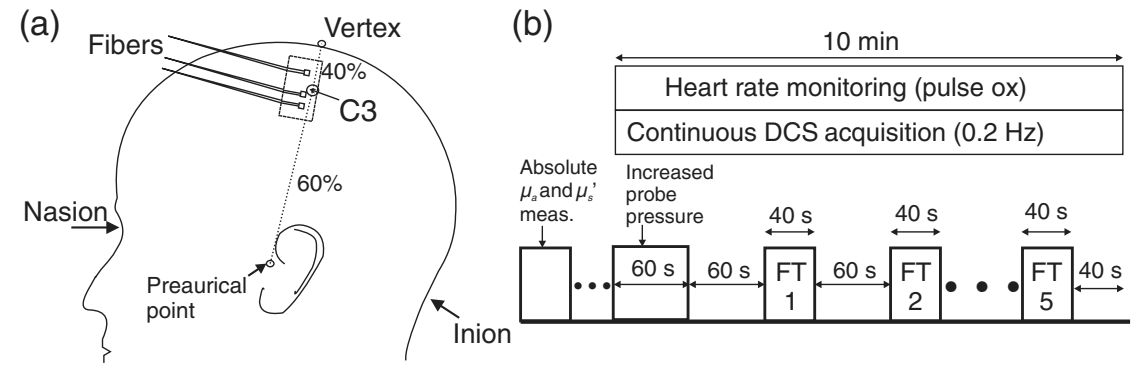

Fig. 7 Cerebral blood flow monitoring during functional activation. (a) To measure the cerebral blood flow response to finger-tapping (FT), a DCS optical probe $\left(\rho_{l}=3.0, \rho_{s}=1.0 \mathrm{~cm}\right)$ was secured over the hand knob area of the motor cortex, which is slightly anterior to the C3 position in the 10-20 EEG coordinate system. ${ }^{98}$ The C3 position lies 2/5 of the distance between the vertex and the preaurical point (i.e., 3 to $4 \mathrm{~cm}$ down from vertex), and the vertex is the halfway point on the curve connecting the nasion to the inion (17 to $18 \mathrm{~cm}$ from nasion). The subject's heart rate was also monitored with a pulse oximeter. (b) Schematic showing the timeline of the FT measurement. The subject did five blocks of FT (i.e., tapping all four fingers of the right hand against the thumb) at $3 \mathrm{~Hz}$. Prior to $\mathrm{FT}$, baseline absolute optical properties were measured over the probe location depicted in (a) (see main text); the probe pressure was temporarily increased by gently pressing down on the probes with the palm of the hand.

(Sec. 6.2), the subject's baseline optical properties over the motor cortex [Fig. 7(a)] were measured first. Then, the cerebral blood flow response to finger-tapping was monitored with a DCS optical probe $\left(\rho_{l}=3.0 \mathrm{~cm}, \rho_{s}=1.0 \mathrm{~cm}\right)$. The probe was secured over the motor cortex [Fig. 7(a)] with double-sided medical tape (3M 1509, Converters Inc., Huntingdon Valley, Pennsylvania) and an all-cotton elastic bandage wound around the head. The subject's heart rate was also monitored in parallel with a pulse oximeter (Radical TM, Masimo, Irvine, California) attached to the subject's left index finger.

With the probe in place, an initial pressure calibration (Fig. 3) was performed by gently pressing down on the probe with the palm of the hand, as depicted in Fig. 7(b). Then the subject executed five finger-tapping trials consisting of $40 \mathrm{~s}$ intervals of finger-tapping separated by $60 \mathrm{~s}$ rest intervals [Fig. 7(b)]. During finger-tapping, the subject tapped all four fingers of the right hand against the thumb at $3 \mathrm{~Hz}$, in time with an audible cuing signal provided by a metronome.

\section{Results}

\subsection{Validation with Simulated Data}

We tested the pressure modulation algorithms (Figs. 3 and 5) on the simulated data sets described in Sec. 6.1. The cerebral blood flow and tissue absorption changes computed with the pressure modulation algorithms are compared to the semi-infinite blood flow and tissue absorption changes (Sec. 2) in Fig. 8. Note that in the flow pressure modulation algorithm, we utilized 42 delay times ranging from $\tau=0.2$ to $\tau=35 \mu$ s to evaluate Eq. (13) for $\Delta F_{c}$. All delay times satisfied the limit $g_{2}^{0}\left(\tau, \rho_{l}\right)>1.25$.

Since the short separation measurements predominantly sample the extracerebral layer, the semi-infinite hemodynamic changes obtained from the short-separation data agree well with the true extracerebral hemodynamic changes. For the 14 extracerebral changes spanning 50 to $100 \%$ in Fig. 8(b), the percent error in the fractional extracerebral flow change is $-1.1 \pm$ $0.7 \%$ (mean $\pm \mathrm{SD}$ ), and the percent error in the fractional extracerebral absorption change [Fig. 8(d)] is $1 \pm 6 \%$. The long separation measurements, however, sample both cerebral and extracerebral tissues.
Substantial signal contamination from the extracerebral tissues induced significant errors in the long-separation semiinfinite estimates of cerebral flow and absorption (Fig. 8). The pressure modulation algorithms successfully filtered much of this extracerebral contamination from the measured signals and led to the recovery of cerebral hemodynamics with higher accuracy (Fig. 8). More quantitatively, the percent error [mean $\pm \mathrm{SD}$ across the 14 cerebral changes spanning -50 to $100 \%$ in Fig. 8(a)] in the cerebral flow computed with the pressure algorithm was $-6 \pm 11 \%$, while the percent error in cerebral blood flow computed with the semi-infinite model $\left(\rho_{l}\right.$ separation) was $-68 \pm 2 \%$. Similarly, percent errors in the cerebral absorption [Fig. 8(c)] computed with the pressure algorithm and with the semi-infinite model are $-8 \pm 24$ and $-82 \pm 5 \%$, respectively.

Interestingly, comparing Figs. 8(a) and 8(b) with Figs. 8(c) and $8(\mathrm{~d})$, it is evident that the semi-infinite DOS/NIRS calculation is less sensitive to the brain than the semi-infinite DCS calculation. ${ }^{25,66}$ For example, the semi-infinite DOS/NIRS calculation ( $\rho_{l}$ separation) in Fig. 8(d) more closely resembles the extracerebral changes $(-3 \pm 5 \%)$ than the semi-infinite DCS calculation in Fig. 8(b) $(-34 \pm 2 \%)$.

\subsection{Validation with Graded Scalp Ischemia}

As described in Sec. 6.2, we acquired DCS measurements on the forehead of a healthy adult volunteer during graded scalp ischemia. The subject's baseline cerebral flow, extracerebral flow, and extracerebral layer thickness obtained from the calibration stage of the pressure modulation algorithm were $F_{c}^{0}=$ $4.53 \times 10^{-8} \mathrm{~cm}^{2} / \mathrm{s}, F_{e c}^{0}=2.23 \times 10^{-9} \mathrm{~cm}^{2} / \mathrm{s}$, and $\ell=1.35 \mathrm{~cm}$, respectively [Fig. 9(a)]. Further, the baseline DCS photon count rates for the long and short separations were 35 and $170 \mathrm{kHz}$, and the measured baseline optical properties over the forehead at $\lambda=785 \mathrm{~nm}$ are $\mu_{a}^{0}=0.12$ and $\mu_{s}^{\prime 0}=8 \mathrm{~cm}^{-1}$. We then monitored cerebral blood flow at several different probe pressures against the head using the DCS pressure modulation algorithm and the semi-infinite model [Fig. 9(b)].

The extracerebral blood flow determined from applying the semi-infinite model to the short-separation data decreased steeply with increasing probe pressure, until it was close to zero at $P=40 \mathrm{~mm} \mathrm{Hg}$. Importantly, the long-separation 

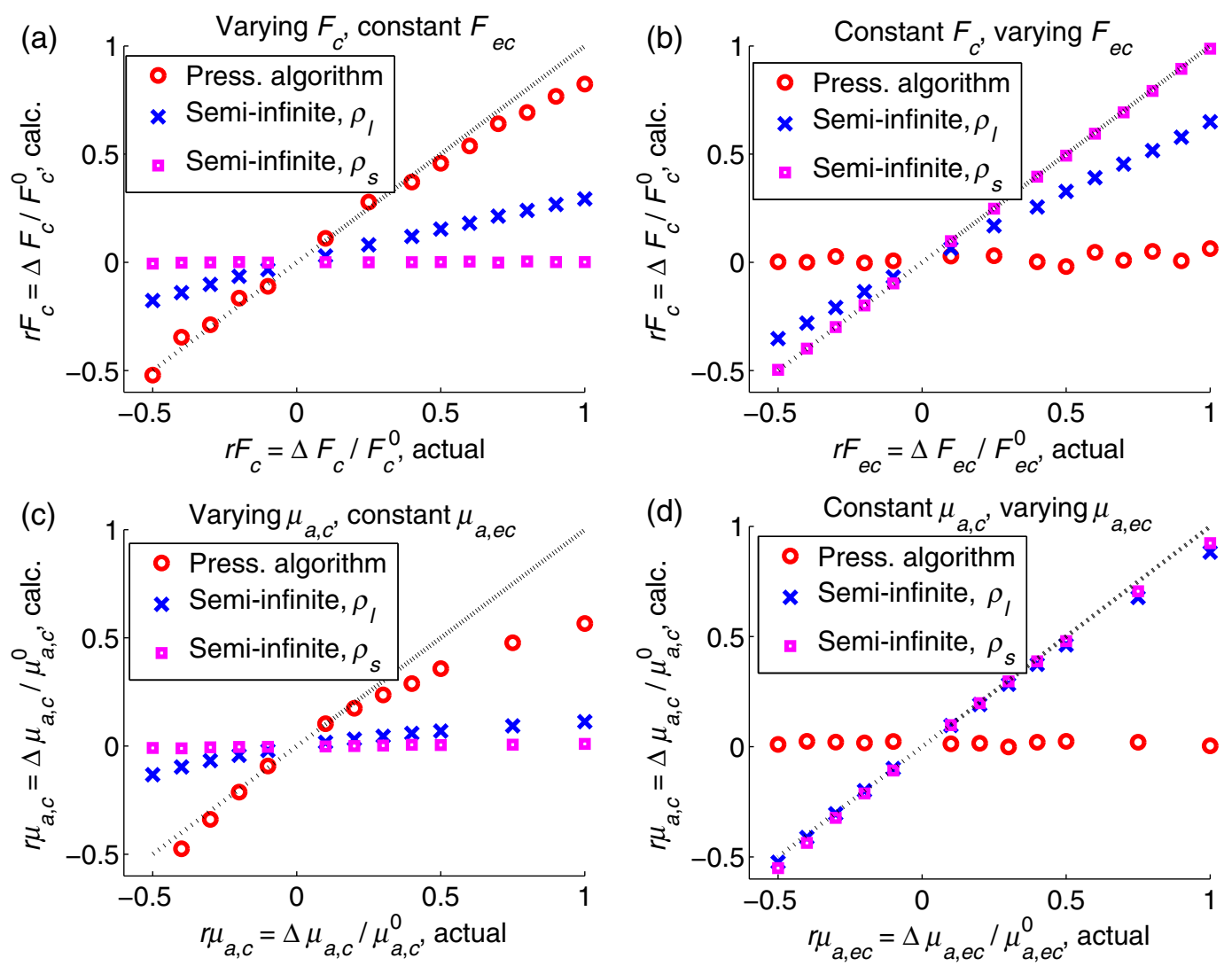

Fig. 8 The DCS and DOS/NIRS pressure modulation algorithms (Figs. 3 and 5) were utilized to calculate cerebral blood flow and tissue absorption changes from simulated measurements on the head acquired at long and short separations of $\rho_{l}=3 \mathrm{~cm}$ and $\rho_{s}=0.7 \mathrm{~cm}$ (see Sec. 6.1). These pressure algorithm results are compared with the homogeneous semi-infinite model estimates of blood flow and tissue absorption computed from the long-separation and the short-separation data. (a) Calculated fractional cerebral blood flow changes plotted against the actual cerebral blood flow change in DCS simulated data set 1 (i.e., extracerebral blood flow remains constant). (b) Calculated fractional cerebral flow changes plotted against the actual extracerebral blood flow change in DCS simulated data set 2 (i.e., cerebral blood flow remains constant). (c) Calculated fractional cerebral absorption changes plotted against the actual cerebral absorption change in DOS/NIRS data set 1 (i.e., extracerebral absorption remains constant). (d) Calculated fractional cerebral absorption changes plotted against the actual extracerebral absorption change in DOS/NIRS data set 2 (i.e., cerebral absorption remains constant).

semi-infinite estimate of cerebral blood flow also decreased substantially with increasing probe pressure, though not as severely as the extracerebral flow. This apparent change in cerebral flow is due to extracerebral contamination in the long-separation signal from the pressure-induced extracerebral flow changes. The DCS pressure modulation algorithm, however, successfully filtered the extracerebral contamination from the long-separation signal; the computed cerebral flow was not affected by probe pressure changes.

Note that for the calculations in this section, the formulation of Sec. 3.4 with multiple delay-times [i.e., 40 delay-times spanning 0.4 to $17.6 \mu \mathrm{s}$ that satisfy $\left.g_{2}^{0}\left(\tau, \rho_{l}\right)>1.25\right]$ for the DCS pressure modulation algorithm was used to obtain the red curve in Fig. 9(b). Further, pressure-induced extracerebral absorption changes, determined from the short-separation signal intensity changes via Eq. (17), were incorporated into the computation of cerebral flow [e.g., Eq. (26)]. Note also that increasing the probe pressure from baseline to $40 \mathrm{~mm} \mathrm{Hg}$ decreased $\mu_{a, e c}$ by $25 \%$; cerebral flow monitoring with the DCS pressure modulation algorithm wherein constant absorption is assumed [i.e., Eq. (15)] resulted in an erroneously calculated increase in cerebral flow of $10 \%$ at $40 \mathrm{~mm} \mathrm{Hg}$.

\subsection{Validation with In Vivo Finger-Tapping Data}

In the second in vivo test, we used the DCS pressure modulation algorithm (Fig. 3) to measure the cerebral flow increase induced by the finger-tapping task in a healthy volunteer (Sec. 6.3). The measured baseline optical properties over the motor cortex at $\lambda=785 \mathrm{~nm}$ were $\mu_{a}^{0}=0.12$ and $\mu_{s}^{\prime 0}=8 \mathrm{~cm}^{-1}$, the baseline DCS photon count rates for the long and short separations were 18 and $140 \mathrm{kHz}$, and the baseline heart rate was $72 \mathrm{bpm}$.

In the calibration stage of this measurement, probe pressure was increased by manually pressing down on the probe with the palm of the hand instead of using a blood pressure cuff wrapped around the head. The subject's baseline cerebral flow, extracerebral flow, and extracerebral layer thickness obtained from the two-layer fit were $F_{c}^{0}=1.95 \times 10^{-8} \mathrm{~cm}^{2} / \mathrm{s}, F_{e c}^{0}=3.08 \times$ $10^{-9} \mathrm{~cm}^{2} / \mathrm{s}$, and $\ell=1.05 \mathrm{~cm}$, respectively [Fig. 10(a)]. The average cerebral flow, extracerebral flow, and heart rate responses induced by finger-tapping $(N=5$ trials $)$ are plotted against time in Fig. 10(b). For comparison, the average semiinfinite flow response for the long separation is also plotted. Notice that the cerebral flow rapidly increases to a steadystate value of $30 \%$ within $5 \mathrm{~s}$ of the start of finger-tapping. The 

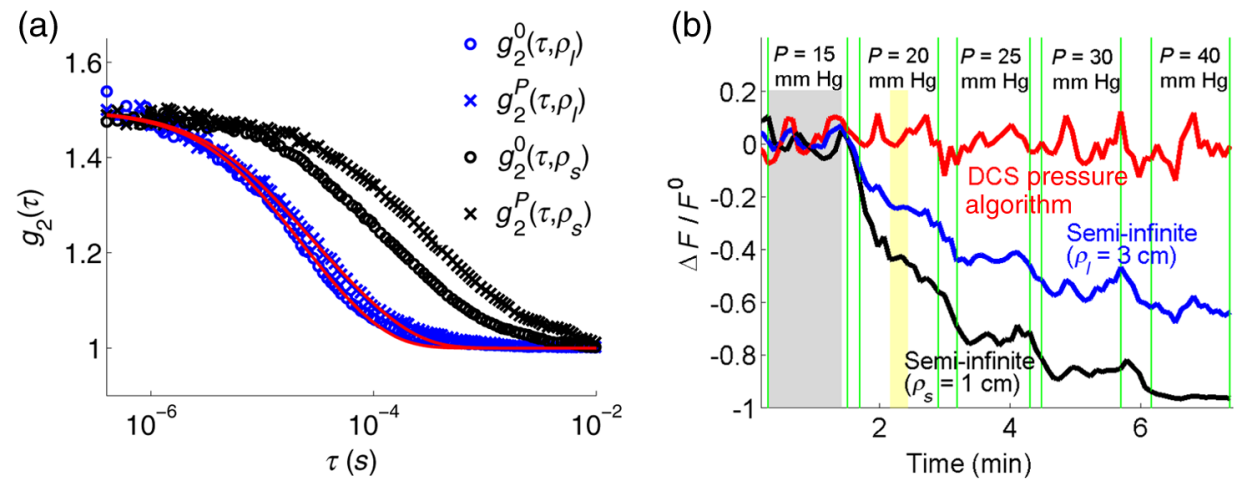

Fig. 9 DCS measurements were acquired on the forehead of a healthy adult volunteer at multiple probe pressures against the head (15 to $40 \mathrm{~mm} \mathrm{Hg}$ ). The optical probe [Fig. 4(c)] consisted of one long sourcedetector separation $\left(\rho_{I}=3 \mathrm{~cm}\right)$ and two short source-detector separations $\left(\rho_{s}=1 \mathrm{~cm}\right)$. (a) Measured intensity autocorrelation curves employed in the calibration stage of the probe pressure modulation algorithm (Fig. 3) plotted against delay-time $\tau . g_{2}^{0}\left(\tau, \rho_{l}\right)$ and $g_{2}^{0}\left(\tau, \rho_{s}\right)$ are the temporally averaged signals across the gray shaded region of (b) (i.e., at $P=15 \mathrm{~mm} \mathrm{Hg} ; N=13$ autocorrelation curves), and $g_{2}^{P}\left(\tau, \rho_{l}\right)$ and $g_{2}^{P}\left(\tau, \rho_{s}\right)$ are the temporally averaged signals across the yellow shaded region of (b) (i.e., at $P=20 \mathrm{~mm} \mathrm{Hg} ; N=4$ autocorrelation curves). The solid red lines indicate the simultaneous two-layer fit of $g_{2}^{0}\left(\tau, \rho_{l}\right)$ and $g_{2}^{P}\left(\tau, \rho_{l}\right)$ for the baseline parameters $F_{c}^{0}, F_{e c}^{0}$, and $\ell$. Note that the two constraints for this fit are $F_{c}^{P}=F_{c}^{0}$ and $F_{e c}^{P} / F_{e c}^{0}=0.57$ [latter constraint obtained from $g_{2}^{0}\left(\tau, \rho_{s}\right)$ and $g_{2}^{P}\left(\tau, \rho_{s}\right)$ via semi-infinite methods]. The extracted baseline parameters from the two-layer fit are $F_{c}^{0}=4.53 \times 10^{-8} \mathrm{~cm}^{2} / \mathrm{s}, F_{e c}^{0}=2.23 \times 10^{-9} \mathrm{~cm}^{2} / \mathrm{s}$, and $\ell=1.35 \mathrm{~cm}$. (b) Temporal fractional flow changes computed with the DCS pressure modulation algorithm and computed with semi-infinite techniques. These fractional flow curves are smoothed via a moving average window of size 3 frames $(15 \mathrm{~s})$. Notice that the cerebral blood flow change computed with the DCS pressure algorithm is not affected by the extracerebral changes induced from varying probe pressure.

extracerebral flow increase, however, is more gradual; its timedependence roughly corresponds to the delayed heart rate increase due to finger-tapping. As expected, the long-separation semi-infinite flow change lies between the cerebral flow change computed with the DCS pressure modulation algorithm [i.e., 38 delay-times spanning 0.4 to $14.4 \mu \mathrm{s}$ were used to evaluate
Eq. (15)] and the extracerebral flow change computed from the short-separation measurements [Fig. 10(b)]. The percent deviation during finger-tapping (mean $\pm \mathrm{SD}$ across seven measurements) between the fractional cerebral flow change computed with the pressure algorithm and that computed with the long-separation semi-infinite model is $25 \pm 19 \%$.
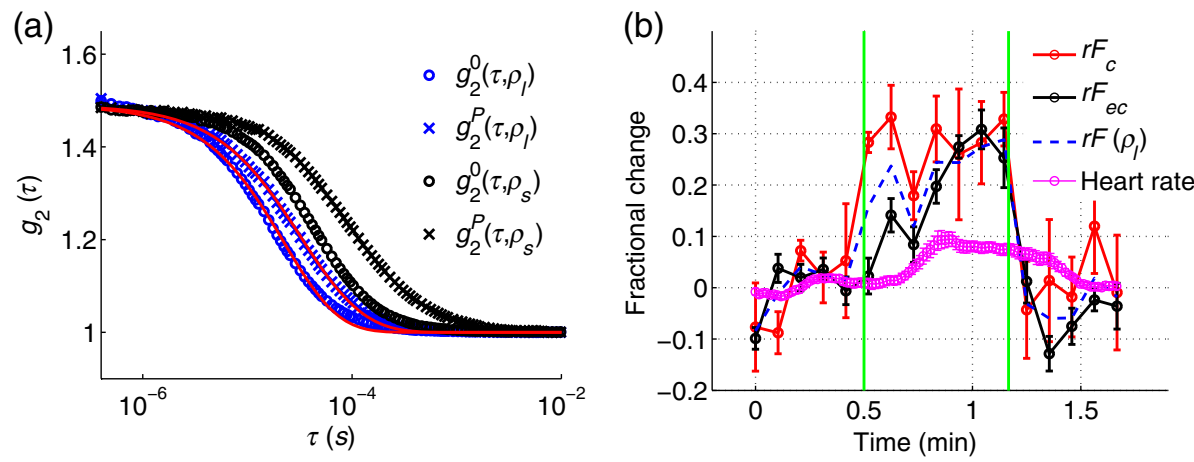

Fig. 10 DCS measurements at one long source-detector separation $\left(\rho_{l}=3 \mathrm{~cm}\right)$ and one short sourcedetector separation $\left(\rho_{s}=1 \mathrm{~cm}\right)$ were acquired over the motor cortex of a healthy adult volunteer while he performed FT [Fig. 7(a)]. (a) Measured intensity autocorrelation curves employed in the calibration stage of the probe pressure modulation algorithm (Fig. 3) plotted against delay-time $\tau$. These curves are temporally averaged signals across the $60 \mathrm{~s}$ (i.e., $N=10$ autocorrelation curves) baseline and increased probe pressure intervals indicated in Fig. $7(\mathrm{~b})$. The solid red lines indicate the simultaneous two-layer fit of $g_{2}^{0}\left(\tau, \rho_{l}\right)$ and $g_{2}^{P}\left(\tau, \rho_{l}\right)$ for the baseline parameters $F_{c}^{0}, F_{e c}^{0}$, and $\ell$, given the constraints that $F_{c}^{P}=$ $F_{c}^{0}$ and that $F_{e c}^{P} / F_{e c}^{O}=0.44$ (latter constraint obtained from $g_{2}^{0}\left(\tau, \rho_{s}\right)$ and $g_{2}^{P}\left(\tau, \rho_{s}\right)$ via semi-infinite methods). The extracted baseline parameters from the two-layer fit are $F_{c}^{0}=1.95 \times 10^{-8} \mathrm{~cm}^{2} / \mathrm{s}$, $F_{e C}^{0}=3.08 \times 10^{-9} \mathrm{~cm}^{2} / \mathrm{s}$, and $\ell=1.05 \mathrm{~cm}$. (b) Measured $\mathrm{FT}$ functional responses (mean $\pm \mathrm{SE}$ across $N=5$ trials) for cerebral blood flow $\left(r F_{c}=\Delta F_{c} / F_{c}^{0}\right)$, extracerebral blood flow $\left(r F_{e c}=\Delta F_{e c} / F_{e c}^{0}\right)$, and heart rate plotted against time. The $\mathrm{FT}$ stimulus was between the two green vertical lines. Here, $r F_{c}$ was computed with the DCS pressure modulation algorithm [Eq. (15)], $r F_{e c}$ was determined by applying semi-infinite methods to the short-separation signal (Sec. 2), and the heart rate was measured with a pulse oximeter on the finger. Further, the blue dashed line $\left[r F\left(\rho_{l}\right)\right]$ is the mean flow response computed from applying the semi-infinite model to the long-separation signal. 


\section{Discussion}

Superficial tissue contamination in optical monitoring of cerebral hemodynamics is a well known issue in the DOS/NIRS community, and several methods have been proposed to isolate the cerebral component in the DOS/NIRS signal. Many of these methods assume statistical independence of superficial and cerebral signals, such as adaptive filtering, ${ }^{38}$ principal component/ independent component analysis, ${ }^{27,32,99}$ state space modeling, ${ }^{33,100}$ and general linear models. ${ }^{26,32,37}$ The justification for this assumption in brain mapping applications is that superficial signals in the scalp arise from systemic effects that are damped by cerebral autoregulation in the brain. Thus, the systemic superficial signals are independent from the local activation signals in the brain. However, as noted in Sec. 1, cerebral autoregulation is impaired in brain diseases such as ischemic stroke. Alternative approaches for filtering superficial tissue contamination include tomographic imaging, ${ }^{54,55,101}$ time-resolved measurements, ${ }^{41,64,65,102}$ and two-layer models. ${ }^{57-63}$

The main result of the present paper is a novel implementation of the two-layer model that utilizes two source-detector separations and probe pressure modulation in order to optically monitor cerebral blood flow (Fig. 3). The two-layer modified Beer-Lambert law for flow is employed to linearly relate DCS signal changes to changes in cerebral and extracerebral blood flow [Eq. (7)]. Further, a patient-specific initial pressure calibration of the measurement substantially improves the tractability of flow monitoring with the two-layer model by reducing the number of free parameters in the model. A priori anatomical information, though helpful, is not required in this pressure modulation algorithm.

In our in vivo tests of graded scalp ischemia (Fig. 9) and finger-tapping (Fig. 10), we did not use any a priori anatomical information. Further, unlike with tomographic imaging and blind source separation analysis, the two-layer model approach does not require a large number of optodes, which permits small area optical probes that are easier to integrate with other monitoring devices in clinical care applications requiring long-term continuous monitoring. Our optical probe for the in vivo tests [Fig. 4(c)] had four optodes. Finally, the linearity of the twolayer modified Beer-Lambert law greatly facilitates long-term continuous real-time monitoring of cerebral blood flow. An analogous pressure modulation algorithm for cerebral absorption monitoring with DOS/NIRS is also introduced in Fig. 5; it represents an extension of the two-layer formulation ${ }^{57}$ of Fabbri et al. to include pressure modulation.

Although the two-layer model is a big simplification of the true head geometry, it is still effective in filtering extracerebral contamination, as we demonstrated in our graded scalp ischemia and finger-tapping tests. Cerebral blood flow calculated with the homogeneous semi-infinite model significantly depended on probe pressure, but the two-layer pressure modulation algorithm calculation of cerebral flow [Eq. (26)] did not (Fig. 9). Further, in our finger-tapping test, the pressure modulation algorithm successfully separated the fast cerebral blood flow increase due to brain activation from the more gradual flow increase due to systemic effects, such as heart rate (Fig. 10).

We measured a steady-state increase in cerebral blood flow from finger-tapping of 30\% [Fig. 10(b)]. This increase is on the low side compared to other published measurements, but is not unreasonable. Durduran et al. measured a mean cerebral blood flow increase of $39 \pm 10 \%$ from finger-tapping $(3 \mathrm{~Hz}){ }^{59}$ Ye et al. measured a $54 \pm 11 \%$ cerebral blood flow increase from finger-tapping $(2 \mathrm{~Hz})$ with arterial spin labeling MRI, ${ }^{103}$ and Kastrup et al. measured a $101 \pm 24 \%$ cerebral blood flow increase from finger-tapping $(3 \mathrm{~Hz})$ with a FAIR MRI technique. ${ }^{104}$ We suspect that our optical probe may not have been perfectly centered over the finger-tapping hand knob (i.e., the finger area of the motor cortex), which is a little less than $2 \mathrm{~cm}$ diameter in size. ${ }^{105}$ The EEG 10-20 system (Fig. 7) only roughly identifies the hand knob location, and we sometimes found it challenging to find the correct position for probe placement. Importantly, we obtained valuable assistance with probe placement from a neurosurgeon (Dr. David Kung). If the probe is not exactly over the hand knob area, then only part of the sampled cerebral volume will encompass the hand knob area, thus inducing a partial volume error in the recovered cerebral flow change that is not accounted for in the two-layer model. This partial volume error results in an underestimation of the magnitude of the flow increase, which is a possible explanation for our lower than expected measured flow increase.

Also notice that although the extracerebral blood flow in the scalp during finger-tapping increases gradually with the heart rate, the extracerebral blood flow and heart rate finger-tapping responses behave qualitatively differently in the poststimulus interval [Fig. 10(b)]. After finger-tapping, the heart rate remains elevated and gradually returns to baseline, while the extracerebral blood flow rapidly plummets, undershooting and then gradually returning to baseline. Several factors can affect superficial tissue blood flow besides the heart rate, such as blood pressure and skin-specific regulation mechanisms. ${ }^{27,37}$ Kirilina et al. investigated the origin of task-evoked hemodynamic changes in the scalp and found that task-evoked superficial artifacts are co-localized with veins draining the scalp. ${ }^{37}$ The observed post stimulus undershoot in extracerebral blood flow could potentially be explained by an increase in scalp venous pressure induced by arterial vasoconstriction following cessation of finger-tapping.

Another interesting aspect of the pressure modulation algorithm is its estimation of the extracerebral layer thickness and baseline cerebral and extracerebral flow indices [Figs. 9(a) and 10(a)]. Regrettably, we do not have independent measures of the extracerebral layer thickness in the two adult subjects. Thus, explicit validation of the extracerebral layer thickness estimates is not possible. The pressure calibration estimate of the layer thickness at the forehead for the pressure variation measurement was $\ell=1.35 \mathrm{~cm}$, while the layer thickness estimate over the motor cortex in the finger-tapping measurement for a different subject was $\ell=1.05 \mathrm{~cm}$. Both of these estimates are within the range of layer thicknesses measured by Durduran et al. from anatomical MRI scans, i.e., $\ell=1.20 \pm 0.26 \mathrm{~cm}$ (unpublished).

Furthermore, the pressure calibration estimate of the ratio of cerebral to extracerebral baseline blood flow in the fingertapping measurement was $F_{c}^{0} / F_{e c}^{0}=6.3$, which is consistent with positron emission tomography measurements in healthy adults. ${ }^{106}$ The estimate of this ratio for the graded scalp ischemia measurement was $F_{c}^{0} / F_{e c}^{0}=20.3$. This ratio is on the high side, but it can be at least partially explained by the probe pressure being a non-negligible $15 \mathrm{~mm} \mathrm{Hg}$ during the baseline state [Fig. 9(b)]. At a probe pressure of $15 \mathrm{~mm} \mathrm{Hg}$, the extracerebral blood flow is substantially lower than it would be normally, which is reflected by the higher than normal cerebral to extracerebral flow ratio estimate. Importantly, we have demonstrated that pressure calibration can be done successfully by simply pressing down on the probe with the palm of the hand 
[Fig. 10(a)]. This easy approach should help facilitate its implementation in clinical settings. Note that our pressure algorithm does not require knowledge of the quantitative pressure being applied to the probe. All that is required is a non-negligible pressure increase to induce a non-negligible extracerebral flow change.

Earlier we discussed how the formulation of the pressure algorithm utilizing Eq. (13) (Fig. 3) is sensitive to correlation noise (Sec. 3.4). For large signals and/or long temporal averaging times, this formulation is effective (Fig. 8), but these luxuries are not always available for cerebral measurements. In our in vivo tests, the correlation noise was sometimes too severe for Eq. (13). In these cases, we used Eqs. (15) and (26) instead, which are more robust to correlation noise. Further, we recommend using multiple delay-times in evaluating these equations for the cerebral flow change to reduce sensitivity to noise. In our in vivo tests, we utilized all delay-times wherein $g_{2}^{0}\left(\tau, \rho_{l}\right)>1.25$ ( $\sim 40$ delay-times).

Noise is less of an issue for the DOS/NIRS pressure algorithm formulation (Fig. 5), because multimode detection fibers offer increased signal strength. During the pressure calibration stage, it is important to ensure that the source-detector separations remain fixed when probe pressure is increased. In our flexible probe, there was a tendency for the source-detector separations to change slightly when pressing down on the probe, and the signal changes were dominated by separation changes rather than extracerebral absorption changes. Note that the DCS measurement is generally less sensitive to these small changes in separation than DOS/NIRS. However, this source-detector separation issue is not a show-stopping challenge: we expect source-detector separations should not change with pressure modulation in a suitably designed probe (e.g., a rigid probe).

\section{Conclusion}

We have introduced and demonstrated a novel DCS pressure modulation algorithm that isolates cerebral blood flow without using a priori anatomical information. The algorithm successfully removed extracerebral artifacts in cerebral measurements of graded scalp ischemia and finger-tapping in human subjects. Thus, the approach should be useful for filtering superficial tissue signal contamination in real-time cerebral blood flow monitoring. An analogous pressure modulation algorithm for DOS/NIRS was introduced, and we demonstrated its ability to filter superficial tissue contamination in simulations of cerebral blood oxygenation monitoring. Together these developments should lead to improvements in the fidelity of cerebral hemodynamic data based on optical signals.

\section{Appendix A: Cerebral Blood Flow Monitoring Pressure Modulation Algorithm when Tissue Optical Properties Vary}

Recall that the results derived in Secs. 3.1 and 3.2 are for the special case of constant tissue absorption and tissue scattering. Here, we relax the constant optical property assumption and derive more general expressions for cerebral flow monitoring in the pressure modulation algorithm framework. We first focus on the case wherein tissue absorption is changing while tissue scattering remains constant. We then consider the case wherein both tissue absorption and scattering vary. Finally, we discuss an alternative formulation of the diffuse correlation spectroscopy
(DCS) pressure modulation algorithm in terms of the detected electric field instead of the detected intensity.

\section{A.1 Cerebral Flow Monitoring with Varying Absorption}

The two-layer DCS modified Beer-Lambert law analogues of Eqs. (7) and (8) that include absorption components are ${ }^{66}$

$$
\begin{aligned}
\Delta \mathrm{OD}_{\mathrm{DCS}}^{\text {long }}= & d_{F, c}\left(\tau, \rho_{l}\right) \Delta F_{c}+d_{F, e c}\left(\tau, \rho_{l}\right) \Delta F_{e c} \\
& +d_{a, c}\left(\tau, \rho_{l}\right) \Delta \mu_{a, c}+d_{a, e c}\left(\tau, \rho_{l}\right) \Delta \mu_{a, e c},
\end{aligned}
$$

$\Delta \mathrm{OD}_{\mathrm{DCS}}^{\text {short }}=d_{F, e c}\left(\tau, \rho_{s}\right) \Delta F_{e c}+d_{a, e c}\left(\tau, \rho_{s}\right) \Delta \mu_{a, e c}$,

where the tissue absorption changes $\Delta \mu_{a, c} \equiv \mu_{a, c}-\mu_{a, c}^{0}$ and $\Delta \mu_{a, e c} \equiv \mu_{a, e c}-\mu_{a, e c}^{0}$ can be estimated from DOS/NIRS measurements via Eqs. (17) and (18), and the multiplicative weighting factors $d_{a, c}\left(\tau, \rho_{l}\right) \equiv \partial \mathrm{OD}_{\mathrm{DCS}}^{\text {long, } 0} / \partial \mu_{a, c}, d_{a, e c}\left(\tau, \rho_{l}\right) \equiv \partial \mathrm{OD}_{\mathrm{DCS}}^{\text {long, } 0} /$ $\partial \mu_{a, e c}$, and $d_{a, e c}\left(\tau, \rho_{s}\right) \equiv \partial \mathrm{OD}_{\mathrm{DCS}}^{\text {short }, 0} / \partial \mu_{a, e c}$ can be numerically determined by evaluating the appropriate derivative of the twolayer correlation diffusion solution at the baseline flow levels, tissue optical properties, and extracerebral layer thickness (e.g., Sec. 3.2.2).

For the pressure calibration stage, the analogues of Eqs. (10) and (11) are

$\Delta \mathrm{OD}_{\mathrm{DCS}}^{\text {long }, P}=d_{F, e c}\left(\tau, \rho_{l}\right) \Delta F_{e c}^{P}+d_{a, e c}\left(\tau, \rho_{l}\right) \Delta \mu_{a, e c}^{P}$,

$\Delta \mathrm{OD}_{\mathrm{DCS}}^{\mathrm{short}, P}=d_{F, e c}\left(\tau, \rho_{s}\right) \Delta F_{e c}^{P}+d_{a, e c}\left(\tau, \rho_{s}\right) \Delta \mu_{a, e c}^{P}$,

where $\Delta \mu_{a, e c}^{P} \equiv \mu_{a, e c}^{P}-\mu_{a, e c}^{0}$ is the pressure-induced change in extracerebral tissue absorption. Solving Eqs. (21), (22), (23), and (24) for the cerebral flow change, we obtain

$$
\begin{aligned}
\Delta F_{c}= & \frac{1}{d_{F, c}\left(\tau, \rho_{l}\right)}\left\{\Delta \mathrm{OD}_{\mathrm{DCS}}^{\text {long }}\right. \\
& -d_{a, e c}\left(\tau, \rho_{l}\right) \Delta \mu_{a, e c}-d_{a, c}\left(\tau, \rho_{l}\right) \Delta \mu_{a, c} \\
& -\frac{\Delta \mathrm{OD}_{\mathrm{DCS}}^{\text {long }, P}-d_{a, e c}\left(\tau, \rho_{l}\right) \Delta \mu_{a, e c}^{P}}{\Delta \mathrm{OD}_{\mathrm{DCS}}^{\text {short } P}-d_{a, e c}\left(\tau, \rho_{s}\right) \Delta \mu_{a, e c}^{P}} \\
& \left.\times\left[\Delta \mathrm{OD}_{\mathrm{DCS}}^{\text {short }}-d_{a, e c}\left(\tau, \rho_{s}\right) \Delta \mu_{a, e c}\right]\right\} .
\end{aligned}
$$

An alternative approach more robust to correlation noise (see Sec. 3.4) is to solve Eq. (21) directly for $\Delta F_{c}$, i.e.,

$$
\begin{aligned}
\Delta F_{c}= & \frac{1}{d_{F, c}\left(\tau, \rho_{l}\right)}\left[\Delta \mathrm{OD}_{\mathrm{DCS}}^{\mathrm{long}}-d_{F, e c}\left(\tau, \rho_{l}\right) \Delta F_{e c}\right. \\
& \left.-d_{a, c}\left(\tau, \rho_{l}\right) \Delta \mu_{a, c}-d_{a, e c}\left(\tau, \rho_{l}\right) \Delta \mu_{a, e c}\right]
\end{aligned}
$$

where $\Delta F_{e c}$ is determined from the short-separation measurements via semi-infinite methods, as described in Sec. 3.4. As with Eq. (13), Eqs. (25) and (26) only require measurements at a single $\tau$ for monitoring, but multiple delay-times should be used to ameliorate sensitivity to noise. 


\section{A.2 Cerebral Flow Monitoring with Varying Absorption and Scattering}

If both tissue absorption and scattering vary significantly, then the differential absorption and scattering changes should be directly measured with concurrent frequency-domain or time-domain DOS/NIRS. ${ }^{5,79,80}$ The extensions of Eqs. (21) and (22) for varying tissue scattering are ${ }^{66}$

$$
\begin{aligned}
\Delta \mathrm{OD}_{\mathrm{DCS}}^{\mathrm{long}}= & d_{F, c}\left(\tau, \rho_{l}\right) \Delta F_{c}+d_{F, e c}\left(\tau, \rho_{l}\right) \Delta F_{e c} \\
& +d_{a, c}\left(\tau, \rho_{l}\right) \Delta \mu_{a, c}+d_{a, e c}\left(\tau, \rho_{l}\right) \Delta \mu_{a, e c} \\
& +d_{s, c}\left(\tau, \rho_{l}\right) \Delta \mu_{s, c}^{\prime}+d_{s, e c}\left(\tau, \rho_{l}\right) \Delta \mu_{s, e c}^{\prime}
\end{aligned}
$$

$$
\begin{aligned}
\Delta \mathrm{OD}_{\mathrm{DCS}}^{\text {short }}= & d_{F, e c}\left(\tau, \rho_{s}\right) \Delta F_{e c}+d_{a, e c}\left(\tau, \rho_{s}\right) \Delta \mu_{a, e c} \\
& +d_{s, e c}\left(\tau, \rho_{s}\right) \Delta \mu_{s, e c}^{\prime},
\end{aligned}
$$

where $\Delta \mu_{s, c}^{\prime} \equiv \mu_{s, c}^{\prime}-\mu_{s, c}^{\prime 0}$ and $\Delta \mu_{s, e c}^{\prime} \equiv \mu_{s, e c}^{\prime}-\mu_{s, e c}^{\prime 0}$ are the differential changes from baseline of cerebral and extracerebral tissue reduced scattering, and the scattering weighting factors $d_{s, c}\left(\tau, \rho_{l}\right) \equiv \partial \mathrm{OD}_{\mathrm{DCS}}^{\text {long, } 0} / \partial \mu_{s, c}^{\prime}, d_{s, e c}\left(\tau, \rho_{l}\right) \equiv \partial \mathrm{OD}_{\mathrm{DCS}}^{\text {long }, 0} / \partial \mu_{s, e c}^{\prime}$, and $d_{s, e c}\left(\tau, \rho_{s}\right) \equiv \partial \mathrm{OD}_{\mathrm{DCS}}^{\text {short }, 0} / \partial \mu_{s, e c}^{\prime}$ are determined using the twolayer correlation diffusion solution as described in Sec. 3.2.2. Pressure-induced signal changes from the extracerebral layer are given by

$$
\begin{aligned}
\Delta \mathrm{OD}_{\mathrm{DCS}}^{\text {long }, P}= & d_{F, e c}\left(\tau, \rho_{l}\right) \Delta F_{e c}^{P}+d_{a, e c}\left(\tau, \rho_{l}\right) \Delta \mu_{a, e c}^{P} \\
& +d_{s, e c}\left(\tau, \rho_{l}\right) \Delta \mu_{s, e c}^{\prime P},
\end{aligned}
$$

$$
\begin{aligned}
\Delta \mathrm{OD}_{\mathrm{DCS}}^{\text {short }, P}= & d_{F, e c}\left(\tau, \rho_{s}\right) \Delta F_{e c}^{P}+d_{a, e c}\left(\tau, \rho_{s}\right) \Delta \mu_{a, e c}^{P} \\
& +d_{s, e c}\left(\tau, \rho_{s}\right) \Delta \mu_{s, e c}^{\prime P} .
\end{aligned}
$$

Solving Eqs. (27), (28), (29), and (30) for the cerebral flow change, we obtain

$$
\begin{aligned}
\Delta F_{c}= & \frac{1}{d_{F, c}\left(\tau, \rho_{l}\right)}\left\{\Delta \mathrm{OD}_{\mathrm{DCS}}^{\text {long }}-d_{a, e c}\left(\tau, \rho_{l}\right) \Delta \mu_{a, e c}\right. \\
& -d_{a, c}\left(\tau, \rho_{l}\right) \Delta \mu_{a, c}-d_{s, e c}\left(\tau, \rho_{l}\right) \Delta \mu_{s, e c}^{\prime} \\
& -d_{s, c}\left(\tau, \rho_{l}\right) \Delta \mu_{s, c}^{\prime}-\frac{d_{F, e c}\left(\tau, \rho_{l}\right)}{d_{F, e c}\left(\tau, \rho_{s}\right)} \\
& \left.\times\left[\Delta \mathrm{OD}_{\mathrm{DCS}}^{\text {short }}-d_{a, e c}\left(\tau, \rho_{s}\right) \Delta \mu_{a, e c}-d_{s, e c}\left(\tau, \rho_{s}\right) \Delta \mu_{s, e c}^{\prime}\right]\right\},
\end{aligned}
$$

where

$$
\begin{aligned}
& \frac{d_{F, e c}\left(\tau, \rho_{l}\right)}{d_{F, e c}\left(\tau, \rho_{s}\right)} \\
& =\frac{\Delta \mathrm{OD}_{\mathrm{DCS}}^{\text {long }, P}-d_{a, e c}\left(\tau, \rho_{l}\right) \Delta \mu_{a, e c}^{P}-d_{s, e c}\left(\tau, \rho_{l}\right) \Delta \mu_{s, e c}^{\prime P}}{\Delta \mathrm{OD}_{\mathrm{DCS}}^{\mathrm{short}, P}-d_{a, e c}\left(\tau, \rho_{s}\right) \Delta \mu_{a, e c}^{P}-d_{s, e c}\left(\tau, \rho_{s}\right) \Delta \mu_{s, e c}^{\prime P}} .
\end{aligned}
$$

Alternatively, the variable scattering extension of Eq. (26) derived from solving Eq. (27) for $\Delta F_{c}$ is

$$
\begin{aligned}
\Delta F_{c}= & \frac{1}{d_{F, c}\left(\tau, \rho_{l}\right)}\left[\Delta \mathrm{OD}_{\mathrm{DCS}}^{\mathrm{long}}-d_{F, e c}\left(\tau, \rho_{l}\right) \Delta F_{e c}\right. \\
& -d_{a, c}\left(\tau, \rho_{l}\right) \Delta \mu_{a, c}-d_{a, e c}\left(\tau, \rho_{l}\right) \Delta \mu_{a, e c} \\
& \left.-d_{s, c}\left(\tau, \rho_{l}\right) \Delta \mu_{s, c}^{\prime}-d_{s, e c}\left(\tau, \rho_{l}\right) \Delta \mu_{s, e c}^{\prime}\right]
\end{aligned}
$$

Again, $\Delta F_{e c}$ is determined from short-separation measurements via semi-infinite techniques.

\section{A.3 Electric Field Formulation of DCS Pressure Algorithm}

Finally, note that the DCS pressure modulation algorithm (Fig. 3) assumes that the coherence of the source laser remains constant over time, i.e., the $\beta$ coefficient in the Siegert relation (see Sec. 2) does not change. If this is not the case, then the DCS signal will change from variation of $\beta^{83}$ in addition to varying flow levels. The pressure algorithm does not account for variations in $\beta$. If $\beta$ is changing, then it is much better to use the DCS modified Beer-Lambert law for the electric field autocorrelation function, $g_{1}(\tau) \equiv\left\langle\mathbf{E}^{*}(t) \cdot \mathbf{E}(t+\tau)\right\rangle /\langle I(t)\rangle$, instead of the intensity autocorrelation function, $g_{2}(\tau)$. The electric field equation is exactly analogous to Fig. 3, except that the DCS optical density $\mathrm{OD}_{\mathrm{DCS}} \equiv-\log \left[g_{2}(\tau)-1\right]$ is replaced with the electric field DCS optical density, $\mathrm{OD}_{\mathrm{DCS}, g 1} \equiv-\log \left[g_{1}(\tau)\right]$. In this equation, the Siegert relation is used to convert the measured $g_{2}$ signals to corresponding $g_{1}$ signals, and the $\beta$ coefficient for each data frame is obtained by a fit.

\section{Appendix B: Derivation of Pressure Modulation Algorithm for Cerebral Absorption Monitoring}

In this appendix, we derive the pressure modulation algorithm for cerebral absorption monitoring, i.e., Eq. (18). The first step is to solve Eqs. (16) and (17) for $\Delta \mu_{a, c}$,

$$
\Delta \mu_{a, c}=\frac{1}{L_{c}\left(\rho_{l}\right)}\left[\Delta \mathrm{OD}^{\text {long }}-\frac{L_{e c}\left(\rho_{l}\right)}{L_{e c}\left(\rho_{s}\right)} \Delta \mathrm{OD}^{\text {short }}\right] .
$$

As is the case for DCS blood flow measurements, the key advantage of using probe pressure modulation with DOS/NIRS is that it enables direct measurement of the ratio $L_{e c}\left(\rho_{l}\right) / L_{e c}\left(\rho_{s}\right)$.

In analogy with Sec. 3.2.1, the ratio $L_{e c}\left(\rho_{l}\right) / L_{e c}\left(\rho_{s}\right)$ can be directly measured from differential short and long source-detector separation optical density changes between perturbed and baseline states wherein only the extracerebral absorption is different. ${ }^{57}$ Probe pressure modulation is a simple way to induce controlled extracerebral absorption changes without affecting cerebral absorption. For the case wherein we wish to compare a perturbed state at probe pressure $P$ to a baseline state at probe pressure $P^{0}$, Eqs. (16) and (17) simplify to

$$
\begin{aligned}
& \Delta \mathrm{OD}^{\text {long }, P} \equiv-\log \left[\frac{I^{P}\left(\rho_{l}\right)}{I^{0}\left(\rho_{l}\right)}\right]=L_{e c}\left(\rho_{l}\right) \Delta \mu_{a, e c}^{P}, \\
& \Delta \mathrm{OD}^{\text {short }, P} \equiv-\log \left[\frac{I^{P}\left(\rho_{s}\right)}{I^{0}\left(\rho_{s}\right)}\right]=L_{e c}\left(\rho_{s}\right) \Delta \mu_{a, e c}^{P},
\end{aligned}
$$

where $I^{P}\left(\rho_{l}\right)$ and $I^{P}\left(\rho_{s}\right)$ are the measured intensities at probe pressure $P$, and $\Delta \mu_{a, e c}^{P} \equiv \mu_{a, e c}^{P}-\mu_{a, e c}^{0}$ is the pressure-induced extracerebral absorption change. 
Dividing Eq. (34) by Eq. (35) and then substituting the result into Eq. (33), we obtain Eq. (18).

\section{Appendix C: Instrumentation and Optical Probe}

For in vivo cerebral blood flow monitoring during finger-tapping and probe pressure variation, we used a custom-built DCS instrument. Briefly, two continuous wave, long coherence length (>5 m) $785 \mathrm{~nm}$ lasers (80 mW, DL785-100-3O, CrystaLaser Inc., Reno, Nevada) illuminate the tissue via multimode fibers. Single-mode detection fibers couple diffusive light emerging from tissue to two arrays of four high-sensitivity single photon counting avalanche photodiodes (SPCM-AQ4C, Excelitas, Quebec, Canada) operating in photon counting mode. The outputs from the detection arrays are connected to a multiple- $\tau$ hardware correlator (Correlator.com, Bridgewater, New Jersey) that computes intensity autocorrelation curves in real time using a correlation integration time of $2.5 \mathrm{~s}{ }^{107}$

For interfacing this instrument with the head, we used an optical probe consisting of one long separation, $\rho_{l}=3.0 \mathrm{~cm}$, and two short separations, $\rho_{s}=1.0 \mathrm{~cm}$ (Fig. 11). All four fiber bundles in the probe are terminated with $3 \mathrm{~mm}$ dielectric coated right-angle prisms (PS905-E02-SP, custom, Thorlabs, Newton, New Jersey). The high reflectivity of the prisms (99\%) ensures high light throughput at the skin-probe interface. Further, as illustrated in Fig. 11, the side-firing prism fibers lay in the same plane as the probe head, which facilitates the application of uniform pressure to the top of the probe.

All seven single-mode fibers in the long-separation detection bundle (DL) and one attenuated single-mode fiber in the DS bundle of the optical probe (Fig. 11) were connected to the eight detection channels in the DCS instrument. The seven independent measurements of the intensity autocorrelation function acquired in parallel at the DL probe position were subsequently averaged together to improve signal-to-noise ratio. For the single-mode fiber in the DS bundle, a variable blocking pigtail style fiber optic attenuator (OZ Optics, Ontario, Canada) was

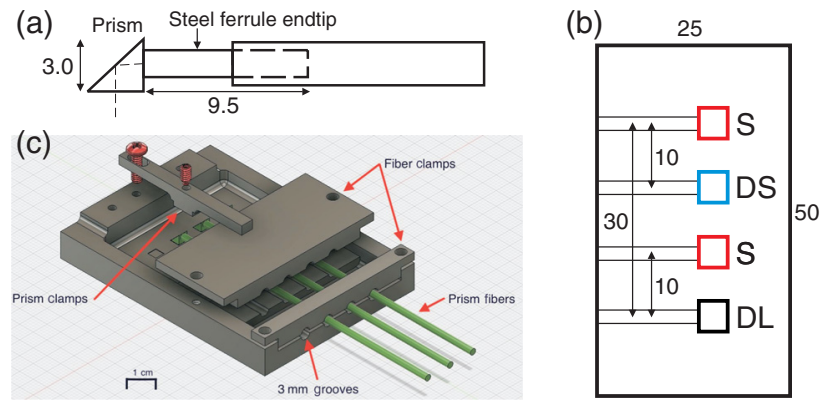

Fig. 11 Schematic of the optical probe used for in vivo DCS measurements (dimensions in $\mathrm{mm}$ ). (a) For conversion of straight ferrule endtip fiber bundles to side-firing prism-coupled fibers, EPO-TEK 353ND epoxy was utilized to glue right-angle prisms (Thorlabs, E03 dielectric coated) to the ferrule endtips. (b) The probe consists of two fused silica multimode source fibers (S; $400 \mu \mathrm{m}$ core/0.22 NA), one longseparation detection bundle (DL) of seven single-mode fibers $(780 \mathrm{HP}$, Thorlabs, $5 \mu \mathrm{m}$ mode field diameter/0.13 NA), and one short-separation detection bundle (DS) of three single-mode fibers (780HP). The termination and bundling of these optical fibers were done by Fiberoptic Systems, Inc. (Simi Valley, California). (c) Schematic of the three-dimensional printed probe mold used for embedding the prism fibers in silicon elastomer at the positions indicated in (b) (see main text). employed to avoid detector saturation. Each $\mathrm{S}$ fiber in the probe was connected to a laser, and the middle $\mathrm{S}$ position fiber was also attenuated (OZ Optics) to avoid detector saturation. During DCS acquisition, multiplexing of the two $S$ positions was achieved by sequentially switching the two DCS lasers on and off with transistor-transistor logic pulses controlled with Labview software (National Instruments, Austin, Texas).

To manufacture the probe, we utilized a three-dimensional printing approach to produce a mold (template) that securely holds the prism fibers at the desired probe positions [Fig. 11(c)]. Specifically, the mold design was made in the Fusion 360 modeling software environment (Autodesk, California) and then printed with VeroClear material using an Objet 500 printer (Stratasys, Minnesota, and Rehovot, Israel). Separately printed prism clamps, with integrated set screws, secured the prism fibers in place along grooves in the mold [Fig. 11(c)]. While the fibers remained fixed in place, a two-part silicone elastomer (VytaFlex-30, Smooth-On, Pennsylvania) was mixed with a black tint (3\% volume fraction; SO-Strong Color Tint, SmoothOn), vacuum degassed, and poured into the mold. Cast as a liquid, the elastomer cures over a time period of $24 \mathrm{~h}$ to form a flexible solid probe head with the prism fibers embedded at the desired positions. Notice that this highly flexible technique can be utilized for making not just flat probes, but also probes with built in curvature that may facilitate measurements on neonates. Finally, if a probe head with higher near-infrared light absorption is desired, we recommend mixing the elastomer with India ink.

\section{Acknowledgments}

We are very grateful to Daniel Licht, John Detre, Michael Mullen, and Steven Schenkel for valuable discussions and assistance with the measurements. Mark Fogel, Yoav Dori, and Kevin Gralewski graciously provided access to their threedimensional printer at the Children's Hospital of Philadelphia and assisted us with printing the optical probe mold. We acknowledge support from the National Institutes of Health (R01-NS060653, NHLBI-HL007915, 8P41-EB015893), the American Heart Association (ABP, 14POST20460161), the Thrasher Pediatric Research Foundation Early Career Award (DRB), the São Paulo Research Foundation (RCM, 2012/ 02500-8, 2013/07559-3), and the Fundaciò Cellex Barcelona (TD). DRB acknowledges support from the Whitaker International Post Doctoral Scholarship administered by the Institute of International Education.

\section{References}

1. R. Mesquita et al., "Direct measurement of tissue blood flow and metabolism with diffuse optics," Philos. Trans. Roy. Soc. A 369(1955), 4390-4406 (2011).

2. T. Durduran and A. G. Yodh, "Diffuse correlation spectroscopy for non-invasive, micro-vascular cerebral blood flow measurement," Neurolmage 85, 51-63 (2014).

3. E. M. Buckley et al., "Diffuse correlation spectroscopy for measurement of cerebral blood flow: future prospects," Neurophotonics 1(1), 011009 (2014).

4. G. Yu, "Diffuse correlation spectroscopy (DCS): a diagnostic tool for assessing tissue blood flow in vascular-related diseases and therapies," Curr. Med. Imaging Rev. 8(3), 194-210 (2012).

5. T. Durduran et al., "Diffuse optics for tissue monitoring and tomography," Rep. Prog. Phys. 73, 076701 (2010).

6. A. Villringer and B. Chance, "Non-invasive optical spectroscopy and imaging of human brain function," Trends Neurosci. 20(10), 435-442 (1997). 
7. H. Obrig and A. Villringer, "Beyond the visible imaging the human brain with light," J. Cereb. Blood Flow Metab. 23(1), 1-18 (2003).

8. M. Smith, "Shedding light on the adult brain: a review of the clinical applications of near-infrared spectroscopy," Philos. Trans. R. Soc. A 369(1955), 4452-4469 (2011).

9. G. Greisen, T. Leung, and M. Wolf, "Has the time come to use nearinfrared spectroscopy as a routine clinical tool in preterm infants undergoing intensive care?" Philos. Trans. R. Soc. A 369(1955), 4440-4451 (2011).

10. F. Scholkmann et al., "A review on continuous wave functional nearinfrared spectroscopy and imaging instrumentation and methodology," Neuroimage 85, 6-27 (2014).

11. S. Lloyd-Fox, A. Blasi, and C. Elwell, "Illuminating the developing brain: the past, present and future of functional near infrared spectroscopy," Neurosci. Biobehav. Rev. 34(3), 269-284 (2010).

12. M. Ferrari and V. Quaresima, "A brief review on the history of human functional near-infrared spectroscopy (FNIRS) development and fields of application," Neuroimage 63(2), 921-935 (2012).

13. D. R. Leff et al., "Assessment of the cerebral cortex during motor task behaviours in adults: a systematic review of functional near infrared spectroscopy (FNIRS) studies," NeuroImage 54(4), 2922-2936 (2011).

14. D. A. Boas and M. A. Franceschini, "Haemoglobin oxygen saturation as a biomarker: the problem and a solution," Philos. Trans. R. Soc. A 369(1955), 4407-4424 (2011).

15. J. P. Culver et al., "Diffuse optical tomography of cerebral blood flow, oxygenation, and metabolism in rat during focal ischemia," J. Cereb. Blood Flow Metab. 23(8), 911-924 (2003).

16. K. S. Lawrence et al., "Measuring cerebral hemodynamics and energy metabolism by near-infrared spectroscopy," in Brain Energy Metabolism, J. Hirrlinger and H. S. Waagepetersen, Eds., Vol. 90, pp. 265-292, Springer, New York (2014).

17. B. K. Siesjö, "Pathophysiology and treatment of focal cerebral ischemia: Part I: pathophysiology," J. Neurosurg. 77(2), 169-184 (1992).

18. W.-D. Heiss, "The ischemic penumbra: How does tissue injury evolve?" Ann. N.Y. Acad. Sci. 1268(1), 26-34 (2012).

19. A. J. Wood, T. Brott, and J. Bogousslavsky, "Treatment of acute ischemic stroke," N. Engl. J. Med. 343(10), 710-722 (2000).

20. C. G. Favilla et al., "Optical bedside monitoring of cerebral blood flow in acute ischemic stroke patients during head-of-bed manipulation," Stroke 45(5), 1269-1274 (2014).

21. T. Durduran et al., "Transcranial optical monitoring of cerebrovascular hemodynamics in acute stroke patients," Opt. Express 17(5), 3884-3902 (2009).

22. T. A. Kent, V. M. Soukup, and R. H. Fabian, "Heterogeneity affecting outcome from acute stroke therapy making reperfusion worse," Stroke 32(10), 2318-2327 (2001).

23. P. Zirak et al., "Transcranial diffuse optical monitoring of microvascular cerebral hemodynamics after thrombolysis in ischemic stroke," J. Biomed. Opt. 19(1), 018002 (2014).

24. H. Obrig and J. Steinbrink, "Non-invasive optical imaging of stroke," Philos. Trans. R. Soc. A 369(1955), 4470-4494 (2011).

25. J. Selb et al., "Sensitivity of near-infrared spectroscopy and diffuse correlation spectroscopy to brain hemodynamics: simulations and experimental findings during hypercapnia," Neurophotonics 1(1), 015005 (2014).

26. S. Tak and J. C. Ye, "Statistical analysis of FNIRS data: a comprehensive review," Neuroimage 85, 72-91 (2014).

27. D. A. Boas, A. M. Dale, and M. A. Franceschini, "Diffuse optical imaging of brain activation: approaches to optimizing image sensitivity, resolution, and accuracy," Neuroimage 23, S275-S288 (2004).

28. R. C. Mesquita et al., "Influence of probe pressure on the diffuse correlation spectroscopy blood flow signal: extra-cerebral contributions," Biomed. Opt. Express 4(7), 978-994 (2013).

29. I. Tachtsidis et al., "False positives in functional nearinfrared topography," Adv. Exp. Med. Biol. 645, 307-314 (2009).

30. T. Takahashi et al., "Influence of skin blood flow on near-infrared spectroscopy signals measured on the forehead during a verbal fluency task," Neuroimage 57(3), 991-1002 (2011).

31. S. N. Davie and H. P. Grocott, "Impact of extracranial contamination on regional cerebral oxygen saturation: a comparison of three cerebral oximetry technologies," Anesthesiology 116(4), 834-840 (2012).
32. T. J. Huppert et al., "Homer: a review of time-series analysis methods for near-infrared spectroscopy of the brain," Appl. Opt. 48(10), D280-D298 (2009).

33. L. Gagnon et al., "Further improvement in reducing superficial contamination in NIRS using double short separation measurements," Neuroimage 85, 127-135 (2014).

34. R. B. Saager, N. L. Telleri, and A. J. Berger, "Two-detector corrected near infrared spectroscopy (c-NIRS) detects hemodynamic activation responses more robustly than single-detector nirs," Neuroimage 55(4), 1679-1685 (2011)

35. S. B. Erdoğan, M. A. Yücel, and A. Aıkn, "Analysis of task-evoked systemic interference in FNIRS measurements: insights from FMRI," NeuroImage 87, 490-504 (2014).

36. T. Yamada, S. Umeyama, and K. Matsuda, "Separation of FNIRS signals into functional and systemic components based on differences in hemodynamic modalities," PLoS One 7(11), e50271 (2012).

37. E. Kirilina et al., "The physiological origin of task-evoked systemic artefacts in functional near infrared spectroscopy," Neuroimage 61(1), 70-81 (2012).

38. Q. Zhang, G. E. Strangman, and G. Ganis, "Adaptive filtering to reduce global interference in non-invasive NIRS measures of brain activation: how well and when does it work?" Neuroimage 45(3), 788-794 (2009).

39. L. B. Goldstein, "Should antihypertensive therapies be given to patients with acute ischaemic stroke?" Drug Safety 22(1), 13-18 (2000).

40. S. L. Dawson, R. B. Panerai, and J. F. Potter, "Serial changes in static and dynamic cerebral autoregulation after acute ischaemic stroke," Cerebrovasc. Dis. 16(1), 69-75 (2003).

41. J. Steinbrink et al., "Determining changes in NIR absorption using a layered model of the human head," Phys. Med. Biol. 46(3), 879 (2001).

42. T. Yamada, S. Umeyama, and K. Matsuda, "Multidistance probe arrangement to eliminate artifacts in functional near-infrared spectroscopy," J. Biomed. Opt. 14(6), 064034 (2009).

43. Y.-K. Liao and S.-H. Tseng, "Reliable recovery of the optical properties of multi-layer turbid media by iteratively using a layered diffusion model at multiple source-detector separations," Biomed. Opt. Express 5(3), 975-989 (2014).

44. J. Ripoll et al., "Recovery of optical parameters in multiple-layered diffusive media: theory and experiments," J. Opt. Soc. Am. A 18(4), 821-830 (2001)

45. A. Liemert and A. Kienle, "Light diffusion in n-layered turbid media: frequency and time domains," J. Biomed. Opt. 15(2), 025002 (2010).

46. L. Wang, S. L. Jacques, and L. Zheng, "MCML-Monte Carlo modeling of light transport in multi-layered tissues," Comput. Methods Programs Biomed. 47(2), 131-146 (1995).

47. F. Jaillon et al., "Diffusing-wave spectroscopy from head-like tissue phantoms: influence of a non-scattering layer," Opt. Express 14(22), 10181-10194 (2006).

48. Q. Liu and N. Ramanujam, "Scaling method for fast Monte Carlo simulation of diffuse reflectance spectra from multilayered turbid media," J. Opt. Soc. Am. A 24(4), 1011-1025 (2007).

49. I. Seo et al., "Perturbation and differential Monte Carlo methods for measurement of optical properties in a layered epithelial tissue model," J. Biomed. Opt. 12(1), 014030 (2007).

50. S. L. Jacques, "Coupling 3D Monte Carlo light transport in optically heterogeneous tissues to photoacoustic signal generation," Photoacoustics 2(4), 137-142 (2014).

51. G. Strangman, M. A. Franceschini, and D. A. Boas, "Factors affecting the accuracy of near-infrared spectroscopy concentration calculations for focal changes in oxygenation parameters," Neuroimage 18(4), 865-879 (2003)

52. D. Boas et al., "Three dimensional Monte Carlo code for photon migration through complex heterogeneous media including the adult human head," Opt. Express 10(3), 159-170 (2002).

53. Q. Fang and D. A. Boas, "Monte Carlo simulation of photon migration in 3D turbid media accelerated by graphics processing units," Opt. Express 17, 20178-20190 (2009).

54. N. M. Gregg et al., "Brain specificity of diffuse optical imaging: improvements from superficial signal regression and tomography," 2010, http://www.ncbi.nlm.nih.gov/pmc/articles/PMC2914577/ (6 July 2015).

55. A. T. Eggebrecht et al., "Mapping distributed brain function and networks with diffuse optical tomography," Nat. Photonics 8, 448-454 (2014). 
56. B. R. White and J. P. Culver, "Phase-encoded retinotopy as an evaluation of diffuse optical neuroimaging," Neuroimage 49(1), 568-577 (2010).

57. F. Fabbri et al., "Optical measurements of absorption changes in twolayered diffusive media," Phys. Med. Biol. 49(7), 1183 (2004).

58. R. B. Saager and A. J. Berger, "Direct characterization and removal of interfering absorption trends in two-layer turbid media," J. Opt. Soc. Am. A 22(9), 1874-1882 (2005).

59. T. Durduran et al., "Diffuse optical measurement of blood flow, blood oxygenation, and metabolism in a human brain during sensorimotor cortex activation," Opt. Lett. 29(15), 1766-1768 (2004).

60. J. Choi et al., "Noninvasive determination of the optical properties of adult brain: near-infrared spectroscopy approach," J. Biomed. Opt. 9(1), 221-229 (2004).

61. B. Hallacoglu, A. Sassaroli, and S. Fantini, "Optical characterization of two-layered turbid media for non-invasive, absolute oximetry in cerebral and extracerebral tissue," PLoS One 8(5), e64095 (2013).

62. O. Pucci, V. Toronov, and K. S. Lawrence, "Measurement of the optical properties of a two-layer model of the human head using broadband near-infrared spectroscopy," Appl. Opt. 49(32), 6324-6332 (2010).

63. L. Gagnon et al., "Investigation of diffuse correlation spectroscopy in multi-layered media including the human head," Opt. Express 16(20), 15514-15530 (2008).

64. L. Gagnon et al., "Double-layer estimation of intra-and extracerebral hemoglobin concentration with a time-resolved system," J. Biomed. Opt. 13(5), 054019 (2008).

65. A. Liebert et al., "Time-resolved multidistance near-infrared spectroscopy of the adult head: intracerebral and extracerebral absorption changes from moments of distribution of times of flight of photons," Appl. Opt. 43(15), 3037-3047 (2004).

66. W. B. Baker et al., "Modified Beer-Lambert law for blood flow," Biomed. Opt. Exp. 5(11), 4053-4075 (2014).

67. A. Yodh and B. Chance, "Spectroscopy and imaging with diffusing light," Phys. Today 48(3), 34-41 (1995).

68. T. J. Farrell, M. S. Patterson, and B. Wilson, "A diffusion theory model of spatially resolved, steady-state diffuse reflectance for the noninvasive determination of tissue optical properties in vivo," Med. Phys. 19(4), 879-888 (1992).

69. D. T. Delpy et al., "Estimation of optical pathlength through tissue from direct time of flight measurement," Phys. Med. Biol. 33(12), 1433 (1988).

70. S. R. Arridge, M. Cope, and D. Delpy, "The theoretical basis for the determination of optical pathlengths in tissue: temporal and frequency analysis," Phys. Med. Biol. 37(7), 1531 (1992).

71. D. Boas, L. Campbell, and A. Yodh, "Scattering and imaging with diffusing temporal field correlations," Phys. Rev. Lett. 75(9), 1855 (1995).

72. D. Boas and A. Yodh, "Spatially varying dynamical properties of turbid media probed with diffusing temporal light correlation," J. Opt. Soc. Am. A 14(1), 192-215 (1997).

73. M. Kohl et al., "Physical model for the spectroscopic analysis of cortical intrinsic optical signals," Phys. Med. Biol. 45, 3749-3764 (2000).

74. S. A. Prahl and S. L. Jacques, "Optical properties spectra," 2012, http:// omlc.ogi.edu/spectra/ (3 April 2014).

75. S. Fantini et al., "Non-invasive optical monitoring of the newborn piglet brain using continuous-wave and frequency-domain spectroscopy," Phys. Med. Biol. 44(6), 1543 (1999).

76. R. C. Haskell et al., "Boundary conditions for the diffusion equation in radiative transfer," J. Opt. Soc. Am. A 11(10), 2727-2741 (1994).

77. D. Hueber et al., "Non-invasive and quantitative near-infrared haemoglobin spectrometry in the piglet brain during hypoxic stress, using a frequency-domain multidistance instrument," Phys. Med. Biol. 46(1), $41(2001)$

78. D. J. Wallace et al., "Results of a 95-subject human clinical trial for the diagnosis of peripheral vascular disease using a near-infrared frequency domain hemoglobin spectrometer," Proc. SPIE 3597, 300-316 (1999).

79. J. B. Fishkin and E. Gratton, "Propagation of photon-density waves in strongly scattering media containing an absorbing semi-infinite plane bounded by a straight edge," J. Opt. Soc. Am. A 10(1), 127-140 (1993).

80. M. S. Patterson, B. Chance, and B. C. Wilson, "Time resolved reflectance and transmittance for the non-invasive measurement of tissue optical properties," Appl. Opt. 28(12), 2331-2336 (1989).
81. V. Jain et al., "Cerebral oxygen metabolism in neonates with congenital heart disease quantified by MRI and optics," J. Cereb. Blood Flow Metab. 34(3), 380-388 (2013).

82. D. C. Lay, Linear Algebra and Its Applications, Addison-Wesley/ Longman Boston, Massachusetts (2000).

83. P. A. Lemieux and D. Durian, "Investigating non-Gaussian scattering processes by using nth-order intensity correlation functions," J. Opt. Soc. Am. A 16(7), 1651-1664 (1999).

84. J. C. Lagarias et al., "Convergence properties of the Nelder-Mead simplex method in low dimensions," SIAM J. Optim. 9(1), 112-147 (1998).

85. M. Hiraoka et al., "A Monte Carlo investigation of optical pathlength in inhomogeneous tissue and its application to near-infrared spectroscopy," Phys. Med. Biol. 38(12), 1859 (1993).

86. R. Panerai, "The critical closing pressure of the cerebral circulation," Med. Eng. Phys. 25(8), 621-632 (2003).

87. A. Kienle and T. Glanzmann, "In vivo determination of the optical properties of muscle with time-resolved reflectance using a layered model," Phys. Med. Biol. 44(11), 2689 (1999).

88. A. Kienle et al., "Noninvasive determination of the optical properties of two-layered turbid media," Appl. Opt. 37(4), 779-791 (1998).

89. S. L. Jacques, "Optical properties of biological tissues: a review," Phys. Med. Biol. 58(11), R37 (2013).

90. P. Wellman et al., "Breast tissue stiffness in compression is correlated to histological diagnosis," 1999, http://www.biorobotics.harvard.edu/ pubs/1999/mechprops.pdf (6 July 2015).

91. H. Hori et al., "The thickness of human scalp: normal and bald," J. Invest. Dermatol. 58(6), 396-399 (1972).

92. D. Irwin et al., "Influences of tissue absorption and scattering on diffuse correlation spectroscopy blood flow measurements," Biomed. Opt. Express 2(7), 1969-1985 (2011).

93. S. Permutt and R. Riley, "Hemodynamics of collapsible vessels with tone: the vascular waterfall," J. Appl. Physiol. 18(5), 924-932 (1963).

94. C. Zhou et al., "Diffuse optical correlation tomography of cerebral blood flow during cortical spreading depression in rat brain," Opt. Express 14(3), 1125-1144 (2006).

95. A. A. Middleton and D. S. Fisher, "Discrete scatterers and autocorrelations of multiply scattered light," Phys. Rev. B 43(7), 5934 (1991).

96. L. Gagnon et al., "Short separation channel location impacts the performance of short channel regression in NIRS," Neuroimage 59(3), 2518-2528 (2012).

97. I. Vogiatzis et al., "Frontal cerebral cortex blood flow, oxygen delivery and oxygenation during normoxic and hypoxic exercise in athletes," J. Physiol. 589(16), 4027-4039 (2011).

98. H. Jasper, "Report of the committee on methods of clinical examination in electroencephalography," Electroencephalogr. Clin. Neurophysiol. 10, 370-375 (1958).

99. S. Patel et al., "Quantification of systemic interference in optical topography data during frontal lobe and motor cortex activation: an independent component analysis," Adv. Exp. Med. Biol. 701, 45-51 (2011).

100. L. Gagnon et al., "Improved recovery of the hemodynamic response in diffuse optical imaging using short optode separations and state-space modeling," Neuroimage 56(3), 1362-1371 (2011).

101. S. G. Diamond et al., "Dynamic physiological modeling for functional diffuse optical tomography," Neuroimage 30(1), 88-101 (2006).

102. J. Selb, D. K. Joseph, and D. A. Boas, "Time-gated optical system for depth-resolved functional brain imaging," J. Biomed. Opt. 11(4), 044008 (2006)

103. F. Q. Ye et al., "Quantitation of regional cerebral blood flow increases during motor activation: a multislice, steady-state, arterial spin tagging study," Magn. Reson. Med. 42(2), 404-407 (1999).

104. A. Kastrup et al., "Changes of cerebral blood flow, oxygenation, and oxidative metabolism during graded motor activation," NeuroImage 15(1), 74-82 (2002).

105. T. Yousry et al., "Localization of the motor hand area to a knob on the precentral gyrus. a new landmark," Brain 120(1), 141-157 (1997).

106. E. Ohmae et al., "Cerebral hemodynamics evaluation by near-infrared time-resolved spectroscopy: correlation with simultaneous positron emission tomography measurements," Neuroimage 29(3), 697-705 (2006).

107. J. Dong et al., "Diffuse correlation spectroscopy with a fast Fourier transform-based software autocorrelator," J. Biomed. Opt. 17(9), 097004 (2012). 
Wesley B. Baker is a postdoctoral researcher in physics and astronomy at the University of Pennsylvania. He graduated with a $\mathrm{PhD}$ in physics at the University of Pennsylvania in May 2015. His research interests include cerebral blood flow and oxygen metabolism monitoring with optical spectroscopy/imaging techniques.

Ashwin B. Parthasarathy is a postdoctoral researcher in physics and astronomy at the University of Pennsylvania. He graduated with a $\mathrm{PhD}$ in biomedical engineering from the University of Texas at Austin in 2010 and was subsequently a postdoctoral researcher at Boston University before moving to Penn in 2012. His overall research goal is the development of optical technology for clinical biomedical applications, focusing on the imaging and monitoring of cerebral blood flow during and after stroke, with laser speckle contrast imaging and diffuse correlation spectroscopy, respectively.

Tiffany S. Ko is an HHMI Interfaces in Imaging doctoral student in the Department of Bioengineering at the University of Pennsylvania. She completed her undergraduate training in electrical engineering at Princeton University in 2009 and received her master's in bioengineering with a focus in translational medicine at the University of California, Berkeley, in 2012. Currently, she works jointly with the Yodh Biomedical Optics Group and Licht Neurovascular Imaging Group at the Children's Hospital of Philadelphia (CHOP). Combining diffuse optical measures of cerebral hemodynamics with MRI analysis, she endeavors to develop noninvasive, quantitative bedside diagnostics of pediatric neurodevelopment and psychomotor function for populations at high risk for hypoxic-ischemic injury.

David R. Busch is a research fellow in the Division of Neurology at $\mathrm{CHOP}$, where his work focuses on noninvasive optical techniques to monitor cerebral autoregulation and metabolism in critically and chronically ill children. His graduate work at the University of Pennsylvania focused on diffuse optical imaging and monitoring of breast cancer. He is currently a Whitaker scholar at ICFO-The Institute of Photonic Sciences, Spain, and has recently been appointed a Fulbright scholar.

Kenneth Abramson is a research engineer in the Yodh lab at the University of Pennsylvania. He completed his undergraduate training in commerce and engineering at Drexel University in 1988, and he specializes in 3D printing of optomechanical components and design of electrical circuits to facilitate biomedical optics research.

Shih-Yu Tzeng is a doctoral student in biomedical optics at the Department of Photonics, National Cheng Kung University. He was a visiting scholar in physics and astronomy at the University of Pennsylvania from October 2014 to April 2015. His research interests include utilizing diffuse optics techniques to measure hemodynamics in superficial tissues (e.g., skin).

Rickson C. Mesquita is an assistant professor of physics at the University of Campinas (UNICAMP, São Paulo, Brazil). He leads a group in biomedical optics with interests focused on designing new instrumentation and developing innovative methods for diffuse optics in biological tissue. He has extensive experience in translating diffuse optical techniques for clinical applications.

Turgut Durduran was trained at the University of Pennsylvania. In 2009, he moved to ICFO-The Institute of Photonic Sciences, Spain, where he leads the medical optics group. His research interests revolve around the use of diffuse light to noninvasively probe tissue function. The group develops new technologies and algorithms and routinely translates them to preclinical, clinical, and industrial applications.

Joel H. Greenberg is a research professor in the Department of Neurology at the University of Pennsylvania. His research interests include hemodynamic and metabolic studies of cerebral ischemia to understand neuronal changes during ischemia and to examine various neuroprotective strategies. He has examined functional activation of cerebral tissue in physiologic and pathophysiologic states in both laboratory animals and humans. He utilizes laser speckle contrast imaging as well as diffuse correlation spectroscopy and diffuse optical spectroscopy in his studies.

David K. Kung is an assistant professor of neurosurgery at the University of Pennsylvania. He has a specific clinical interest in neurovascular diseases and provides a full spectrum of treatments using microsurgical, radiosurgical, and endovascular techniques. In addition, he is actively engaged in the study of cerebral aneurysms, including mechanisms of growth and rupture as well as novel treatments.

Arjun G. Yodh is the James M. Skinner professor of science and the director of the Laboratory for Research on the Structure of Matter (LRSM) at the University of Pennsylvania. His current interests span fundamental and applied questions in condensed matter physics, medical and biophysics, and optical sciences. He has extensive experience utilizing diffuse optics techniques for monitoring and imaging of hemodynamics in living tissues. 